\title{
Espelhos e seções cônicas
}

\author{
Paolino Roberto Rosi
}



SERVIÇO DE PÓS-GRADUAÇÃO DO ICMC-USP

Data de Depósito:

Assinatura:

\title{
Paolino Roberto Rosi
}

\section{Espelhos e seções cônicas}

\author{
Dissertação apresentada ao Instituto de Ciências \\ Matemáticas e de Computação - ICMC-USP, como \\ parte dos requisitos para obtenção do título de \\ Mestre em Ciências - Programa de Mestrado \\ Profissional em Matemática. VERSÃO REVISADA. \\ Área de Concentração: Matemática. \\ Orientador: Prof. Dr. Miguel Vinícius Santini Frasson
}

USP - São Carlos

Fevereiro de 2017 
Ficha catalográfica elaborada pela Biblioteca Prof. Achille Bassi e Seção Técnica de Informática, ICMC/USP, com os dados fornecidos pelo(a) autor(a)

Rosi, Paolino Roberto

R819e Espelhos e seções cônicas / Paolino Roberto Rosi: orientador Miguel Vinícius Santini Frasson. -- São Carlos, 2017.

$56 \mathrm{p}$.

Dissertação (Mestrado - Programa de Pós-Graduação em Mestrado Profissional em Matemática em Rede Nacional) -- Instituto de Ciências Matemáticas e de Computação, Universidade de São Paulo, 2017.

1. Cônicas. 2. Aplicações das cônicas. 3. Propriedade reflexiva das cônicas. 4. Esferas de Dandelin. I. Frasson, Miguel Vinícius Santini, orient. II. Título. 


\section{Paolino Roberto Rosi}

\section{Mirrors and conic sections}

Master dissertation submitted to the Instituto de Ciências Matemáticas e de Computação - ICMCUSP, in partial fulfillment of the requirements for the degree of the Master Program in Professional Master's Program in Mathematics. FINAL VERSION.

Concentration Area: Mathematics.

Advisor: Prof. Dr. Miguel Vinícius Santini Frasson

USP - São Carlos

February 2017 

Dedico este trabalho à minha esposa, Flavia, companheira amada e incentivadora deste projeto, aos meus amados filhos Vinícius e Maria Fernanda, por entenderem a necessidade dos momentos de ausência e aos meus pais que tanto amo Francisco e Edna. 


\section{Agradecimentos}

A Deus por me proporcionar paciência e coragem;

À minha esposa por me auxiliar de diversas maneiras;

Aos meus colegas de turma, companheiros de luta;

E ao Prof. Miguel, pela paciência, dedicação e incentivo. 


\section{Resumo}

Esta pesquisa tem por objetivo investigar as aplicações das cônicas no processo de ensino e aprendizagem, dando uma ampla visão histórica e aplicada do conteúdo em questão. Serão dadas as definições, propriedades, equações e conceitos das cônicas. Classificaremos cônicas a partir de sua equação geral. Apresentaremos as esferas de Dandelin e daremos algumas aplicações das cônicas.

Palavras-chave: cônicas; aplicações das cônicas; propriedade reflexiva das cônicas; esferas de Dandelin. 


\section{Abstract}

This research aims to investigate conic sections in the process of teaching and learning, providing a broad historic and applied view of the subject. It will be discussed definitions, properties, equations and concepts of the conic sections. We classify them from their general equation. We present the Dandelin spheres and provide applications of the conics.

Keywords: conics; applications of the conics; reflexive property of the conics; Dandelin spheres. 


\section{Sumário}

\begin{tabular}{ll}
\hline Introdução & 11
\end{tabular}

$\begin{array}{lll}1 & \text { Surgimento das cônicas } & 13\end{array}$

$\begin{array}{lll}2 & \text { A parábola } & 17\end{array}$

2.1 Definição geométrica . . . . . . . . . . . . . . . . . . 17

2.1 .1 Elementos de uma parábola . . . . . . . . . . . . . . . 18

2.2 Equação reduzida da parábola . . . . . . . . . . . . . . . . . . 19

2.2.1 Parábola com eixo de simetria sobre o eixo $x$, vértice na origem .................... 19

$2.2 .2 \quad$ Parábola com eixo de simetria sobre o eixo $y$, vértice na origem e foco acima do vértice . . . . . . . . . 20

2.2 .3 Parábola com vértice fora da origem e diretriz vertical 21

2.2.4 Parábola com vértice fora da origem e diretriz horizontal 21

2.3 Função quadrática e a parábola . . . . . . . . . . . . . . 22

3 Elipse $\mathbf{2 5}$

3.1 Definição geométrica . . . . . . . . . . . . . . . . . 25

3.1 .1 Elementos de uma elipse . . . . . . . . . . . . . . . . 25

3.2 Equação reduzida . . . . . . . . . . . . . . . . . 26

3.2 .1 Elipse com centro na origem e focos sobre o eixo $x$. . . 26

3.2 .2 Elipse com centro fora da origem e eixo maior paralelo ao eixo $x \ldots \ldots \ldots \ldots . \ldots . \ldots 27$

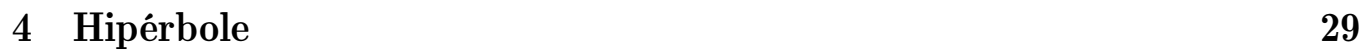

4.1 Definição geométrica . . . . . . . . . . . . . . . . . . . . . . . 29

$4.1 .1 \quad$ Elementos de uma hipérbole . . . . . . . . . . . . . . . 29

4.2 Equação reduzida . . . . . . . . . . . . . . . . . . . 30 
4.2.1 Hipérbole com centro na origem e focos sobre o eixo $x \quad 30$

4.2 .2 Hipérbole com centro na origem e focos sobre o eixo $y$. 32

4.2 .3 Hipérbole com centro fora da origem e eixo real paralelo ao eixo $x \ldots \ldots . \ldots . \ldots . \ldots 33$

4.2.4 Hipérbole com centro fora da origem e eixo real paralelo ao eixo $y$. . . . . . . . . . . . . . . 34

\begin{tabular}{|lll}
5 & Equação geral das cônicas & $\mathbf{3 7}$
\end{tabular}

5.1 Eliminando o termo misto . . . . . . . . . . . . . . . . . . 38

5.2 Eliminando os termos lineares . . . . . . . . . . . . . . . . . . 39

5.3 Exemplos . . . . . . . . . . . . . . . . . . . . 39

$6 \quad$ As esferas de Dandelin $\quad 41$

6.1 Elipse . . . . . . . . . . . . . . . . . . . . . . . . . 41

6.2 Hipérbole . . . . . . . . . . . . . . . . . . . . . . . . . . . . . 42

6.3 Parábola . . . . . . . . . . . . . . . . . . . . . 44 44

\begin{tabular}{|ll|}
\hline Aplicações das cônicas & 47
\end{tabular}

7.1 Espelhos parabólicos . . . . . . . . . . . . . . . . . . . . 47

7.2 Espelhos elípticos . . . . . . . . . . . . . . . . . . . . . . . . . 49

7.3 Espelhos hiperbólicos . . . . . . . . . . . . . . . . . . . . 52

7.4 Sistema de navegação LORAN . . . . . . . . . . . . . . . . . 54 


\section{Introdução}

A presente pesquisa buscou investigar as aplicações das cônicas no processo de ensino e aprendizagem do correspondente conteúdo matemático e pretende auxiliar os professores a dar uma ampla visão histórica e principalmente aplicada do conteúdo em questão. Ao pesquisar sobre o assunto na bibliografia, nota-se o fato de que no documento Proposta Curricular do Estado de São Paulo, disciplina matemática - ensino fundamental, ciclo II e ensino médio (2008), está proposta a organização dos conteúdos contribuindo para a discussão e reflexão, norteando desse modo a prática docente nos diferentes ciclos do ensino; expõe que, para a $3^{\text {a }}$ série do ensino médio, deve haver a noção e aplicação das cônicas, e o que queremos é explorar principalmente as aplicações da parábola, da elipse e da hipérbole, despertando assim o interesse dos alunos, que muitas vezes questionam para que servem determinados conteúdos.

Esse trabalho apresenta-se assim estruturado:

No Capítulo 1, farei uma descrição da história das cônicas e dos grandes pensadores que as estudaram já desde séculos antes de Cristo, tentando fazer uma ligação sobre o que é pesquisar, que pode parecer não muito importante em um primeiro momento mas fica evidente pelas inúmeras aplicações cotidianas.

No Capítulo 2, relatarei sobre a parábola, definindo e mostrando as principais propriedades e conceitos que são estudados no ensino médio, para posteriormente no capítulo final poder tratar algumas de suas aplicações.

No Capítulo 3, tratarei a respeito da elipse, também definindo e mostrando suas principais propriedades que são discutidas no ensino médio, para poder relatar posteriormente no Capítulo 7 algumas de suas aplicações.

No Capítulo 4, discutirei sobre a hipérbole, definindo e mostrando os principais conceitos que são vistos no ensino médio, para dessa forma poder tratar de suas aplicações. 
No Capítulo 5, analisarei uma cônica por sua equação geral, transformando essa equação na forma reduzida, conseguindo assim identificar de qual cônica se trata.

No Capítulo 6, demonstrarei como Germinal Dandelin e Adolphe Quetelet mostraram que a elipse, a hipérbole e a parábola podem ser obtidas seccionando-se um cone duplo por meio de um plano de inclinação variável em relação ao seu eixo.

No Capítulo 7, demonstrarei a propriedade reflexiva das cônicas e exemplificarei esse fato mostrando algumas de suas principais aplicações. O sistema de navegação LORAN também será estudado. 


\section{Capítulo 1}

\section{Surgimento das cônicas}

If I have seen further it is by standing on the shoulders of Giants. (Se vi mais longe, foi por estar de pé sobre ombros de gigantes.)

Isaac Newton.

Nosso principal objetivo no capítulo que se segue é desenvolver um breve panorama histórico acerca dos mais diferentes estudos matemáticos já realizados sobre o tema. Procuraremos sobretudo, apontar e discutir brevemente as origens das cônicas e a trajetória do seu desenvolvimento na longa história da matemática.

Como início deste percurso histórico, gostaríamos de referenciar o importante artigo de Venturi, intitulado "O segundo problema clássico da Geometria: a duplicação do cubo" 19. No artigo, o autor narra um antigo problema com o qual se depararam os atenienses e a sua insatisfatória resposta diante da exigência do Oráculo. Segundo Venturi:

Conta uma lenda que, em 429 a.C., durante o cerco espartano na Guerra do Peloponeso, uma peste dizimou um quarto da população de Atenas, matando inclusive Péricles, e que uma plêiade de sábios fora enviada ao oráculo de Apolo, em Delfos, para inquirir como a peste poderia ser eliminada. O oráculo respondeu que o altar cúbico de Apolo deveria ser duplicado. Os atenienses celeremente dobraram as medidas das arestas do cubo.

Apesar da presteza dos atenienses em obedecer às vontades do deus Apolo, deus da verdade, da ordem e da medida, a solução por eles encontrada não 
era a fiel execução da exigência. O que está errado, pois, a solução de dobrar as arestas do cubo, tendo em vista que ao invés de dobrar o altar, eles octuplicaram o volume do cubo.

A mesma questão de duplicação de um cubo aparece envolvendo um outro problema na antiguidade, dessa vez envolvendo o túmulo do filho do rei Minos. Conforme relata Eves, em "Introdução a história da matemática":

"Há indícios de que o problema da duplicação do cubo possa ter se originado nas palavras de algum poeta grego antigo (talvez Eurípedes), ignorante em matemática, ao descrever a insatisfação do mítico rei Minos com o tamanho do túmulo erguido para seu filho Glauco. Minos ordenou que o tamanho do túmulo fosse dobrado. O poeta fez então Minos aduzir, incorretamente, que isso poderia ser feito dobrando-se cada uma das dimensões do túmulo. Essa falha matemática da parte do poeta levou os geômetras a abraçar o problema de como dobrar um dado sólido mantendo-se sua forma" [5, p.135].

O fato é que não se sabe ao certo o verdadeiro problema de dobrar o volume de um paralelepípedo, seja ele um cubo ou não. O mais interessante, em todas essas histórias é que, finalmente, Menaecmus, por volta de 350 a.C. foi o inventor das curvas parábola, elipse e hipérbole. Tais invenções seriam aquelas empregadas por ele na resolução do problema da duplicação do cubo. Menaecmus obteve, geometricamente, o ponto de interseção da parábola $x^{2}=2 y$ com a hipérbole $x y=1$, a solução é $x=\sqrt[3]{2}$.

Euclides de Alexandria (325 a.C.-265 a.C.), autor do mais antigo livro de matemática ainda utilizado nos dias de hoje, só perdendo em número de edições para a Bíblia, escreveu sobre as seções cônicas em uma de suas obras, intitulada "Cônicas", infelizmente perdida.

Arquimedes, nascido em 287 a.C. na cidade de Siracusa, escreveu entre muitas outras obras o tratado "A Quadratura da Parábola", no qual realizou a grande proeza de mostrar como calcular, pelo método da exaustão, a área de qualquer segmento de parábola, que é a superfície delimitada por um arco de uma parábola e um segmento de reta que intersecta esse arco em dois pontos. Além disso, no seu tratado "Sobre Conoides e Esferoides", Arquimedes estudou elipses, hipérboles e parábolas. O livro possui 32 proposições, e em uma dessas (a Proposição 4), mostra que a área da elipse de semieixos $a$ e $b$ é $\pi a b$, fato desconhecido até então. 
Três gigantes da matemática, Euclides, Arquimedes e Apolônio, todos eles ligados à Universidade de Alexandria, marcaram um período que, mais tarde, seria chamado de Idade de Ouro do contexto do mundo ocidental clássico.

Apolônio nasceu em 262 a.C., na cidade de Perga, conhecido como "o grande geômetra" e faleceu em Alexandria por volta de 190 a.C.. Apolônio fez muitos trabalhos e sabe-se que muitos deles foram perdidos. Ele é mais lembrado, no entanto, pela sua obra-prima "Cônicas", contendo 8 livros, dos quais 7 chegaram até nós, sendo três em árabe e quatro em grego, contendo mais de 480 proposições sobre elipse, hipérbole e parábola. Essa obra foi escrita de forma muito abrangente e excepcional, tanto que trabalhos escritos anteriormente a cerca desse tema, foram praticamente esquecidos pelos matemáticos, interessando talvez aos historiadores mais atentos à história desse tão importante problema matemático. Apolônio foi o primeiro a adotar a termologia elipse, hipérbole e parábola. Segundo Silva em seu artigo "Porque elipse, parábola e hipérbole?" [15], essa terminologia foi inspirada na linguagem pitagórica (VI séc. a.C.), usada pelo também matemático grego para a definição específica de triângulos. Quando os pitagóricos, montando seus problemas, faziam com que uma extremidade de um lado do triângulo (base), coincidisse com uma extremidade de um segmento de reta, eles tinham os casos de parábola, elipse ou hipérbole, se a base fosse igual ao segmento seria uma parábola, quando a base fosse menor que o segmento seria uma elipse e sendo a base maior que o segmento uma hipérbole. Tal termologia é o próprio significado dos termos parábola, elipse e hipérbole: parábola é o mesmo que igual, elipse significa falta, hipérbole quer dizer excesso. Apesar de outros matemáticos já terem utilizado seções de cone para mostrar as cônicas, Apolônio foi o primeiro a utilizar o cone duplo seccionado para a obtenção das cônicas (Figura 1.1).

Deixando para trás a Antiguidade Clássica, temos que referenciar Johannes Kepler, matemático alemão, nascido em 1571 e falecido em 1630, responsável por um excelente e reconhecido trabalho sobre a trajetória elíptica dos planetas. Kepler considerou que o Sol estava em um dos focos da elipse, e partir disso estudou sobre as aplicações óticas das cônicas, e entre outros estudos, foi capaz de demonstrar importantes aplicações às cônicas. No mesmo período, Pierre de Fermat, nascido em 1601 e falecido em 1665, criou as equações cartesianas da reta, da circunferência e as equações simplificadas sobre as cônicas, que visavam pensar como estabelecer que a função do primeiro grau é uma reta e a função do segundo grau é uma parábola. O importante matemático e físico Isaac Newton, nascido em 1643 e falecido em 1727, se- 
ria o responsável por continuar os importantes avanços dos estudos elípticos realizados por Kepler. Newton tornou possível, então, o estudo das cônicas analiticamente.

Apesar de Apolônio ter sido o primeiro a provar que as cônicas podem ser geradas a partir do cone duplo, feito que inscreveu para sempre seu nome na história da matemática, no final do século XVIII dois matemáticos, Germinal Dandelin (1794-1847) e Adolphe Quetelet (1796-1874), encontraram maneiras mais simples e elegantes de fazê-lo, tema conhecido como Esferas de Dandelin, conteúdo esse que será tratado no Capítulo6.

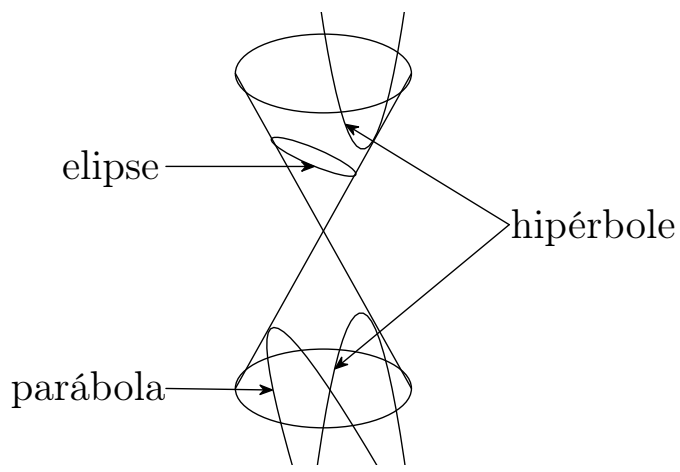

Figura 1.1: Seções cônicas. 


\section{Capítulo 2}

\section{A parábola}

\subsection{Definição geométrica}

A parábola é o lugar geométrico no plano dos pontos que estão equidistantes a uma reta $d$ (diretriz) e a um ponto $F$ (foco) não pertencente a $d$ (Figura 2.1.).

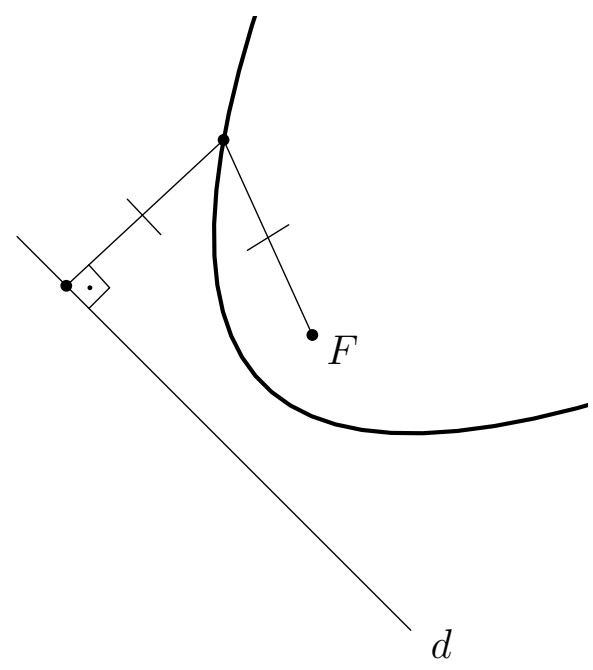

Figura 2.1: Parábola: lugar geométrico dos pontos equidistantes a $d$ e a $F$. 


\subsubsection{Elementos de uma parábola}

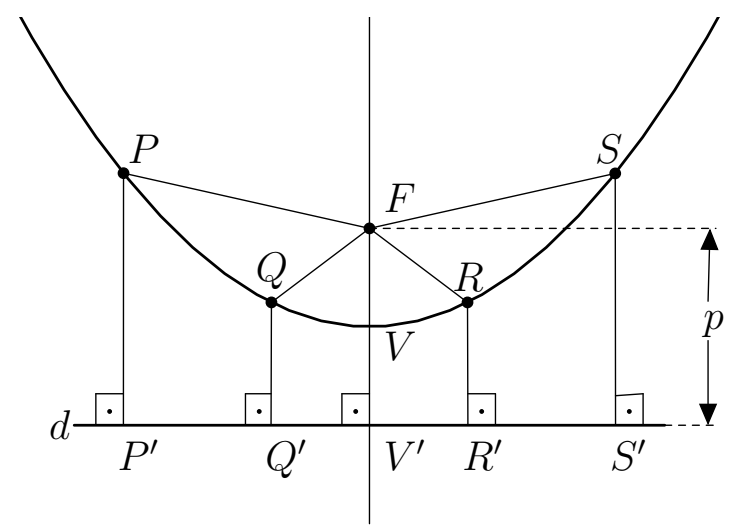

Figura 2.2: Elementos principais de uma parábola.

Pela definição temos na Figura 2.2 que $V F=V V^{\prime}, P F=P P^{\prime}, Q F=$ $Q Q^{\prime}, R F=R R^{\prime}$ e $S F=S S^{\prime}$. E temos como elementos principais, o foco $F$, a diretriz $d$, o vértice $V$, o eixo de simetria é a reta que passa por $V F$, o parâmetro $p$ e a relação notável $V F=\frac{p}{2}$ 


\subsection{Equação reduzida da parábola}

\subsubsection{Parábola com eixo de simetria sobre o eixo $x$, vértice na origem}

Seja $\lambda$ uma parábola com eixo de simetria sobre o eixo $x$ e vértice $V(0,0)$. Pela Figura 2.3 temos que o foco tem coordenadas $F\left(\frac{p}{2}, 0\right)$ e a reta diretriz tem equação $x=-p / 2$. Seja $P(x, y) \in \lambda$ e $P^{\prime}\left(-\frac{p}{2}, y\right)$ a projeção ortogonal de $P$ sobre $d$.

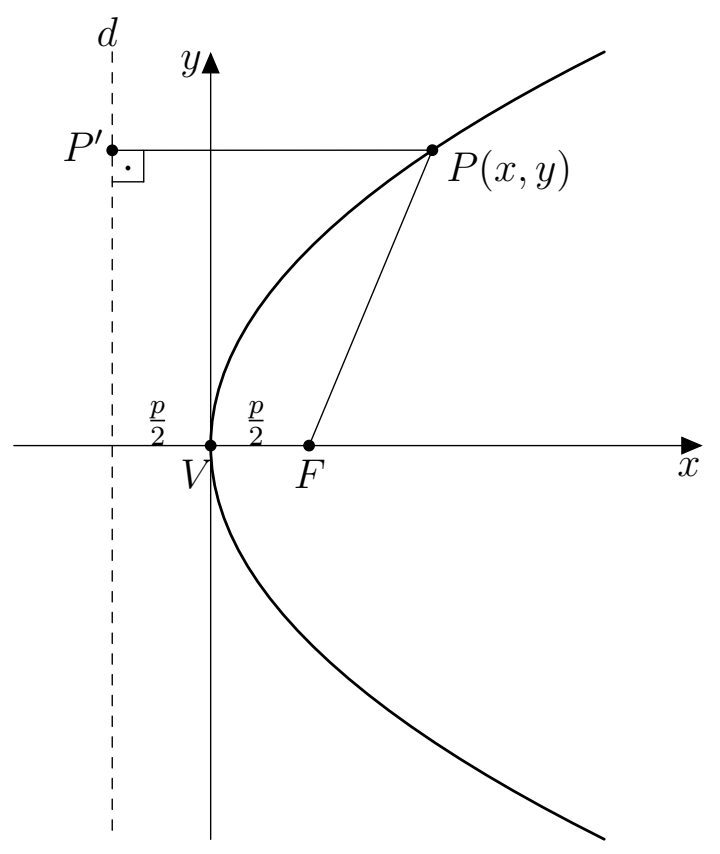

Figura 2.3: Vértice na origem e foco a direita da diretriz.

Como $P \in \lambda$, temos pela definição que $P F=P P^{\prime}$, isto é,

$$
\sqrt{\left(x-\frac{p}{2}\right)^{2}+(y-0)^{2}}=\sqrt{\left(x+\frac{p}{2}\right)^{2}+(y-y)^{2}} .
$$

Elevando ao quadrado membro a membro e desenvolvendo, temos

$$
x^{2}-p x+\frac{p^{2}}{4}+y^{2}=x^{2}+p x+\frac{p^{2}}{4} .
$$


Rearranjando os termos, teremos a equação

$$
y^{2}=2 p x .
$$

$\mathrm{Na}$ verdade, fizemos o caso em que o foco está à direita do vértice. O caso do foco à esquerda do vértice é análogo, resultando na equação reduzida

$$
y^{2}=-2 p x .
$$

\subsubsection{Parábola com eixo de simetria sobre o eixo $y$, vértice na origem e foco acima do vértice}

Seguindo a linha da seção anterior, pela Figura 2.4. temos os pontos $V(0,0)$, $F\left(0, \frac{p}{2}\right), P^{\prime}\left(x,-\frac{p}{2}\right)$ e $P(x, y)$. Como $P$ pertence à parábola, pela definição temos que $P F=P P^{\prime}$ :

$$
\sqrt{(x-0)^{2}+\left(y-\frac{p}{2}\right)^{2}}=\sqrt{(x-x)^{2}+\left(y+\frac{p}{2}\right)^{2}}
$$

Desenvolvendo,

$$
x^{2}+y^{2}-p y+\frac{p^{2}}{4}=y^{2}+p y+\frac{p^{2}}{4} .
$$

Dessa forma, teremos a equação

$$
x^{2}=2 p y .
$$

O caso com foco abaixo do vértice é análogo, levando à equação

$$
x^{2}=-2 p y \text {. }
$$

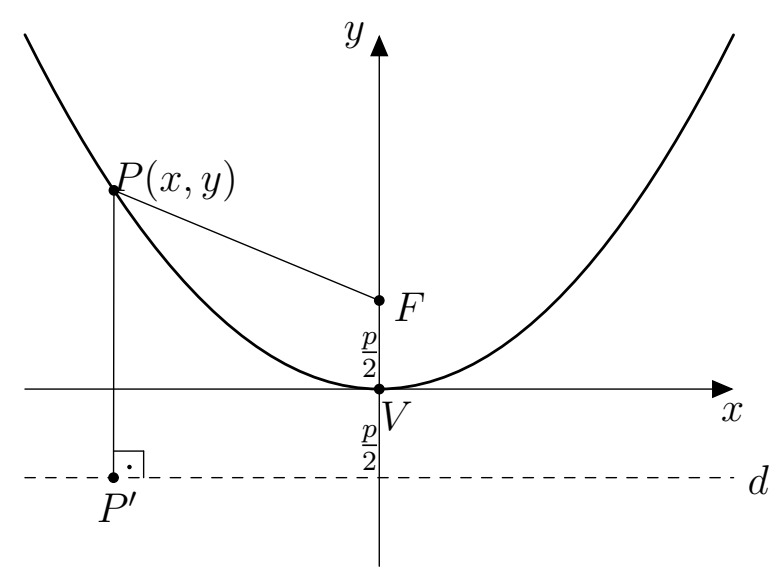

Figura 2.4: Vértice na origem e foco acima da diretriz. 


\subsubsection{Parábola com vértice fora da origem e diretriz vertical}

Quando a reta diretriz é vertical, se o vértice $V\left(x_{0}, y_{0}\right)$ não estiver na origem, podemos reduzir ao caso anterior (Seção 2.2.1) aplicando a todos os pontos a translação que leva o vértice na origem. Assim, o ponto $P$ da parábola terá coordenadas $P\left(x-x_{0}, y-y_{0}\right)$ e portanto (veja a figura 2.5)

$$
\left(y-y_{0}\right)^{2}= \pm 2 p\left(x-x_{0}\right)
$$

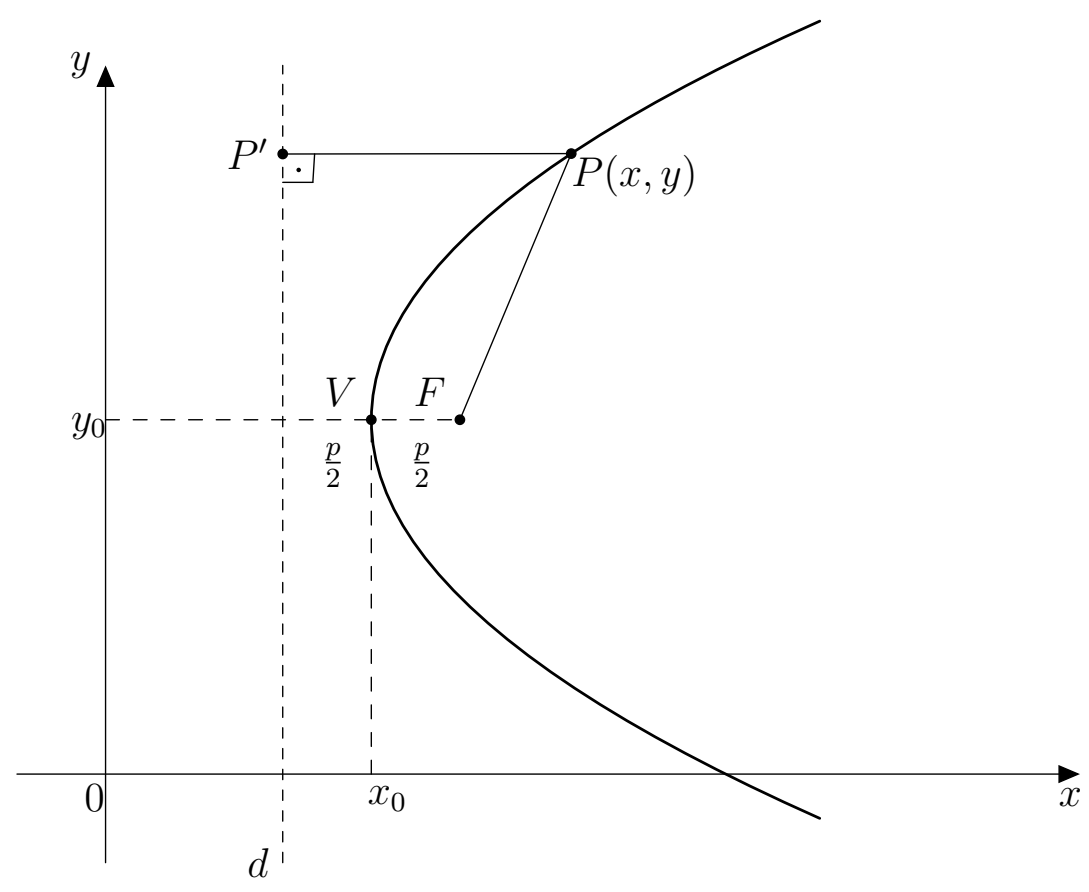

Figura 2.5: Vértice fora da origem e diretriz vertical

\subsubsection{Parábola com vértice fora da origem e diretriz horizontal}

Analogamente ao que fizemos na seção anterior, quando a reta diretriz é horizontal e o vértice $V\left(x_{0}, y_{0}\right)$ não estiver na origem, podemos reduzir ao caso anterior (Seção 2.2.2) aplicando a todos os pontos a translação que leva o vértice na origem. Assim, o ponto $P$ da parábola terá coordenadas $P\left(x-x_{0}, y-y_{0}\right)$ e portanto

$$
\left(x-x_{0}\right)^{2}= \pm 2 p\left(y-y_{0}\right)
$$




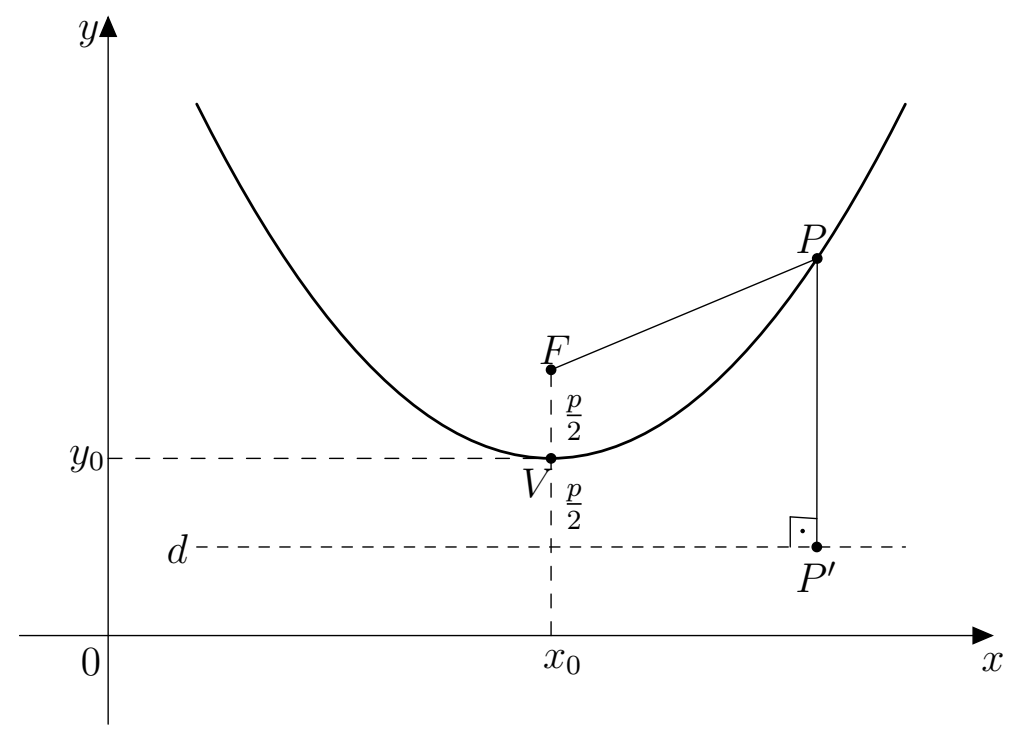

Figura 2.6: Vértice fora da origem e diretriz horizontal.

\subsection{Função quadrática e a parábola}

No currículo do estado de São Paulo inicia-se o ensino da equação do segundo grau no $9^{\mathrm{O}}$ ano do ensino fundamental, dando um enfoque na construção de gráficos por meio de tabelas de pontos, e posteriormente no $1^{\mathrm{O}}$ ano do ensino médio a construção de gráficos começa a ter mais detalhes, como encontrar ponto de máximo ou de mínimo, assim como apresentando ao aluno aquele gráfico como uma parábola, deixando de lado a definição geométrica da parábola até sua abordagem em geometria analítica no $3^{\underline{0}}$ ano do ensino médio. É aceitável que isso aconteça pois de certo modo tem-se que levar em conta o desenvolvimento intelectual do discente perante determinado conteúdo, para que dessa forma possa-se dar sequência a outro tema. No entanto, parece haver uma certa displicência em relação a esse fato, pois quando é definida a parábola nem sempre é feita a conexão entre esses assuntos. E é por esse motivo que nesse momento faço essa ligação, mostrando que a função do segundo 
grau é de fato uma parábola. Para isso, vamos completar quadrados.

$$
\begin{aligned}
y & =a x^{2}+b x+c \\
& =a\left(x^{2}+\frac{b x}{a}+\frac{c}{a}\right) \\
& =a\left(x^{2}+2 \frac{b}{2 a} x+\frac{b^{2}}{4 a^{2}}+\frac{c}{a}-\frac{b^{2}}{4 a^{2}}\right) \\
& =a\left(\left(x+\frac{b}{2 a}\right)^{2}-\frac{b^{2}-4 a c}{4 a^{2}}\right) .
\end{aligned}
$$

Sendo $\Delta=b^{2}-4 a c$, a equação acima pode ser reescrita como

$$
\left(x+\frac{b}{2 a}\right)^{2}=\frac{1}{a}\left(y+\frac{\Delta}{4 a}\right) .
$$

Seguindo a Seção 2.2.4, vemos que (2.1) é a equação da parábola com vértice $\left(-\frac{b}{2 a},-\frac{\Delta}{4 a}\right)$, foco $\left(-\frac{b}{2 a}, \frac{1-\Delta}{4 a}\right)$ e reta diretriz $y=-\frac{1+\Delta}{4 a}$. Além disso, fazendo $y=0 \mathrm{em}$ (2.1) e isolando $x$, chega-se à fórmula das raízes, conhecida como Fórmula de Bhaskara

$$
x=\frac{-b \pm \sqrt{\Delta}}{2 a} .
$$




\section{Capítulo 3}

\section{Elipse}

\subsection{Definição geométrica}

A elipse é o lugar geométrico dos pontos no plano onde a soma das distâncias a certos pontos $F_{1}$ e $F_{2}$ (chamados de focos) é constante.

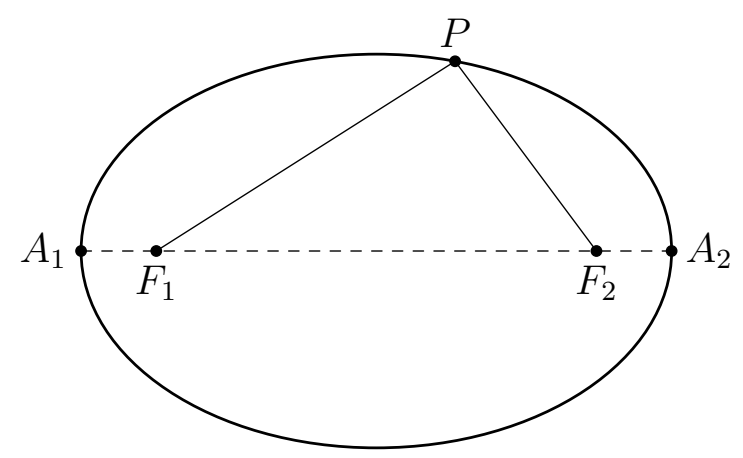

Figura 3.1: $\overline{P F_{1}}+\overline{P F_{2}}=\overline{A_{1} A_{2}}$, para todo $P$ na elipse.

\subsubsection{Elementos de uma elipse}

Temos como elementos da elipse os focos $F_{1}$ e $F_{2}$, o centro $O$, o eixo maior $A_{1} A_{2}$, o eixo menor $B_{1} B_{2}$, a distância focal $2 c$, a medida do eixo maior $2 a$, a medida do eixo menor $2 b$. Além disso, não podemos esquecer da relação pitagórica $a^{2}=b^{2}+c^{2}$. Veja a Figura 3.2 .

A excentricidade da elipse que é um indicador de sua forma, poderia ser definido como o quociente entre a distância focal e a medida do eixo maior 
da elipse, é dada por $e=c / a$. Na elipse, tem-se $0<e<1$. Note que uma circunferência é uma elipse onde os focos degeneram-se para um único ponto, e portanto $e=0$. Também, quanto mais achatada é a elipse, isto é, quando $b$ é próximo de zero, temos que $c$ fica próximo de $a$ e a excentricidade fica próxima de 1.

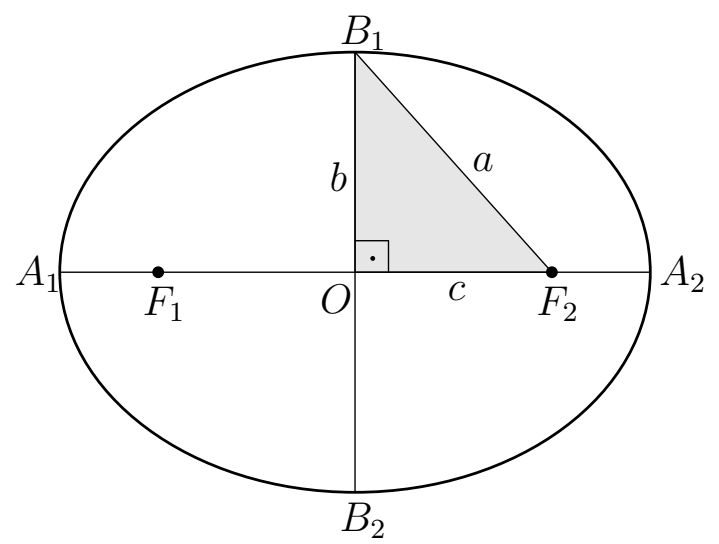

Figura 3.2: Elementos de uma elipse.

\subsection{Equação reduzida}

\subsubsection{Elipse com centro na origem e focos sobre o eixo $x$}

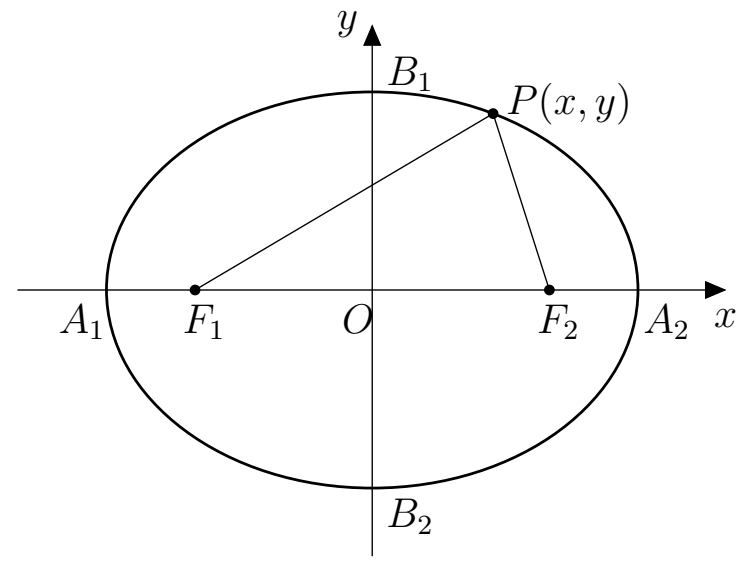

Figura 3.3: Elipse com centro na origem e focos sobre o eixo $x$. 
Seja $\epsilon$ uma elipse com centro $O(0,0)$, focos $F_{1}(-c, 0), F_{2}(c, 0)$ e extremos $A_{1}(-a, 0), A_{2}(a, 0), B_{1}(0, b), B_{2}(0,-b)$. Seja $P(x, y) \in \epsilon$. Pela definição, temos que $P F_{1}+P F_{2}=2 a$. Com o auxílio da Figura 3.3 , vemos que

$$
\begin{gathered}
\sqrt{(x+c)^{2}+(y-0)^{2}}+\sqrt{(x-c)^{2}+(y-0)^{2}}=2 a \\
\sqrt{(x+c)^{2}+y^{2}}=2 a-\sqrt{(x-c)^{2}+y^{2}}
\end{gathered}
$$

Elevando membro a membro ao quadrado, temos

$$
\begin{gathered}
(x+c)^{2}+y^{2}=4 a^{2}-4 a \sqrt{(x-c)^{2}+y^{2}}+(x-c)^{2}+y^{2} \\
x^{2}+2 x c+c^{2}+y^{2}=4 a^{2}-4 a \sqrt{(x-c)^{2}+y^{2}}+x^{2}-2 x c+c^{2}+y^{2} \\
4 x c=4 a^{2}-4 a \sqrt{(x-c)^{2}+y^{2}} \\
a \sqrt{(x-c)^{2}+y^{2}}=a^{2}-x c
\end{gathered}
$$

Elevando membro a membro ao quadrado novamente e desenvolvendo

$$
\begin{gathered}
a^{2} x^{2}-2 a^{2} x c+a^{2} c^{2}+a^{2} y^{2}=a^{4}-2 a^{2} x c+x^{2} c^{2} \\
a^{2} x^{2}-c^{2} x^{2}+a^{2} y^{2}=a^{4}-a^{2} c^{2} \\
\left(a^{2}-c^{2}\right) x^{2}+a^{2} y^{2}=a^{2}\left(a^{2}-c^{2}\right)
\end{gathered}
$$

Substituindo $\left(a^{2}-c^{2}\right)$ por $b^{2}$, pois $a^{2}=b^{2}+c^{2}$,

$$
b^{2} x^{2}+a^{2} y^{2}=a^{2} b^{2}
$$

Dividindo membro a membro por $a^{2} b^{2}$, teremos a equação reduzida da elipse

$$
\frac{x^{2}}{a^{2}}+\frac{y^{2}}{b^{2}}=1
$$

No caso em que o eixo maior da elipse fica sobre o eixo $y$, basta trocar os papéis de $x$ e $y$ para obtermos uma equação reduzida análoga.

\subsubsection{Elipse com centro fora da origem e eixo maior paralelo ao eixo $x$}

Caso o centro $\left(x_{0}, y_{0}\right)$ da elipse $\epsilon$ não esteja na origem, a mudança de variáveis $x^{\prime}=x-x_{0}$ e $y^{\prime}=y-y_{0}$, que é uma translação que leva $\left(x_{0}, y_{0}\right)$ na origem, 
leva a elipse $\epsilon$ numa outra elipse $\epsilon^{\prime}$ com as mesmas medidas e centro na origem no sistema de coordenadas $\left(x^{\prime}, y^{\prime}\right)$. Então,

$$
\frac{x^{\prime 2}}{a^{2}}+\frac{y^{\prime 2}}{b^{2}}=1
$$

Desfazendo a mudança, temos a equação da elipse para este caso:

$$
\frac{\left(x-x_{0}\right)^{2}}{a^{2}}+\frac{\left(y-y_{0}\right)^{2}}{b^{2}}=1
$$

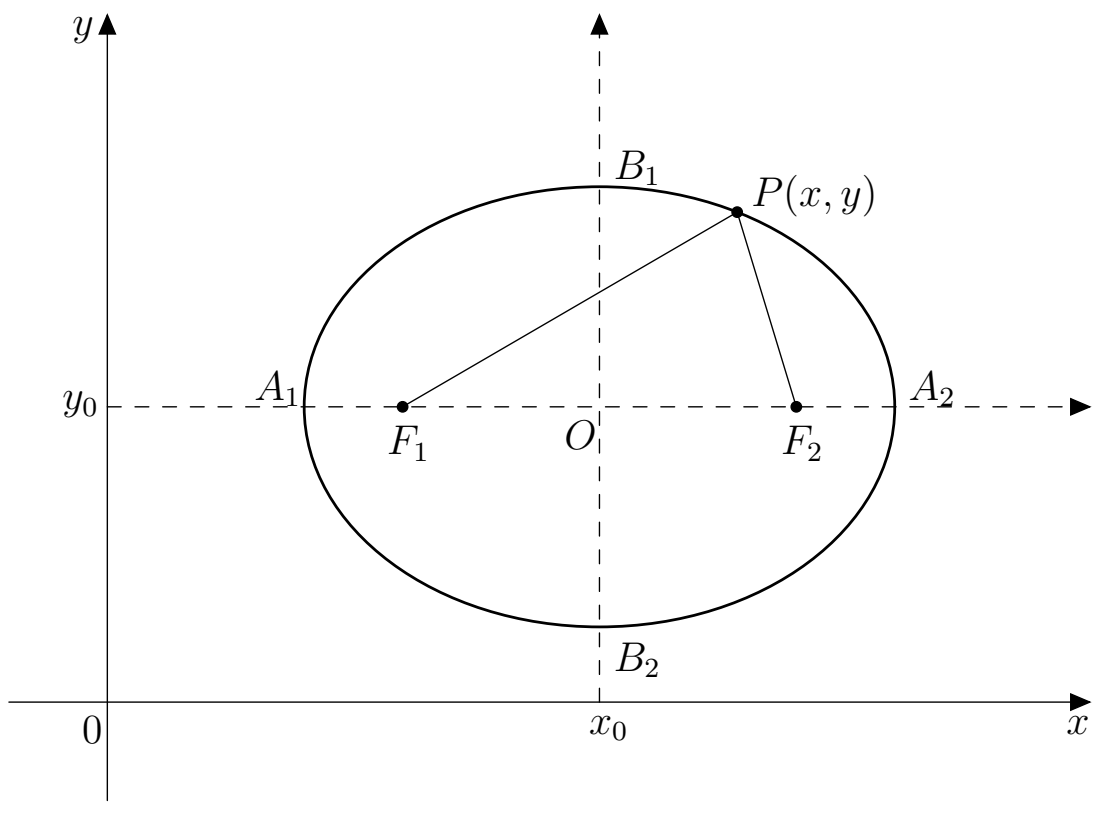

Figura 3.4: Elipse com centro fora da origem e eixo maior paralelo ao eixo $x$ 


\section{Capítulo 4}

\section{Hipérbole}

\subsection{Definição geométrica}

A hipérbole é o lugar geométrico dos pontos do plano tais que o módulo da diferença das distâncias a dois pontos $F_{1}$ e $F_{2}$ (focos) seja constante. Veja a a Figura 4.1 .

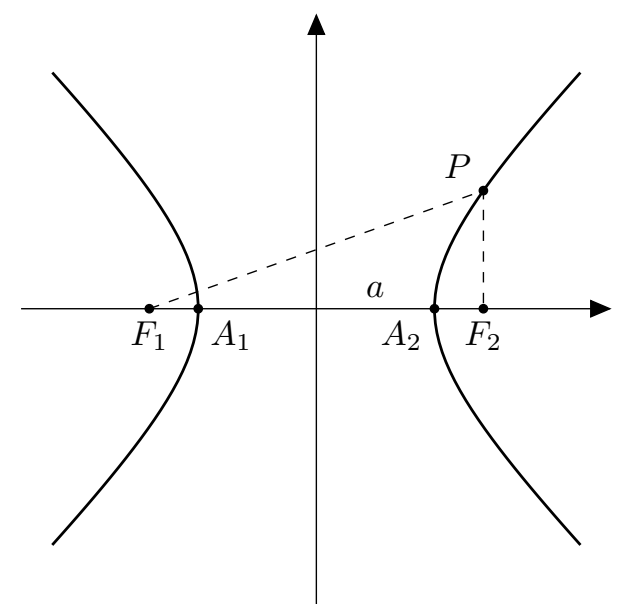

Figura 4.1: Hipérbole: $\left|P F_{1}-P F_{2}\right|=\left|A_{1} F_{1}-A_{1} F_{2}\right|=\left|A_{2} F_{1}-A_{2} F_{2}\right|=2 a$.

\subsubsection{Elementos de uma hipérbole}

Temos como elementos da hipérbole os focos $F_{1}$ e $F_{2}$, o centro $O$, o eixo transverso ou eixo real $A_{1} A_{2}$, o eixo imaginário $B_{1} B_{2}$, a distância focal $2 c$, 
a medida do eixo real $2 a$, a medida do eixo imaginário $2 b$ e a excentricidade $c / a$, além da importante relação pitagórica $c^{2}=a^{2}+b^{2}$.

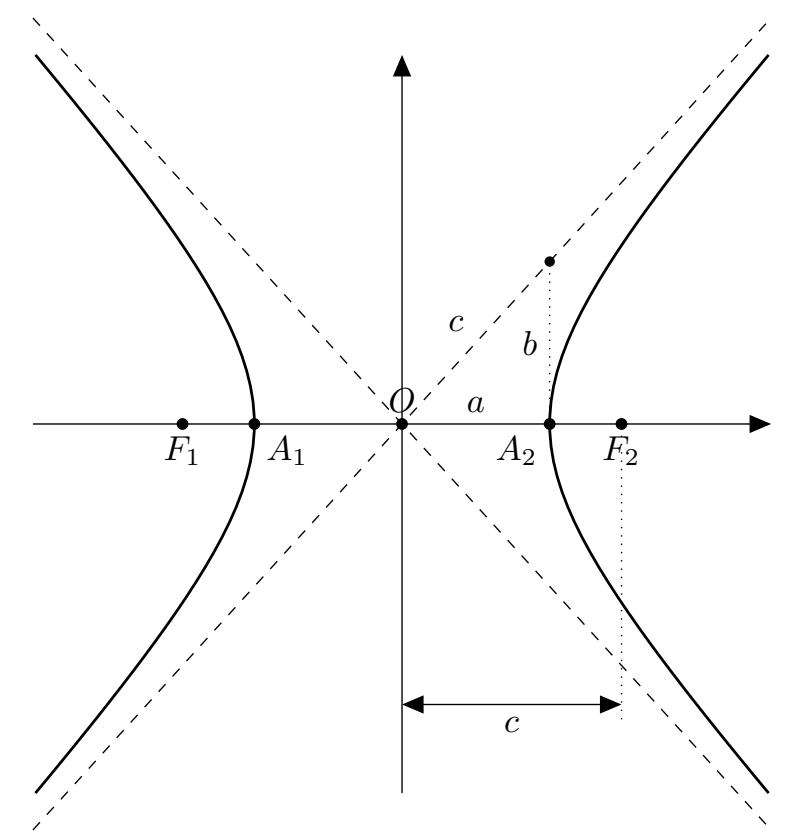

Figura 4.2: Elementos de um hipérbole: Focos $F_{1}, F_{2}$, centro $O$, distância focal $2 c$ e eixo transverso $A_{1} A_{2}=2 a$. As retas assíntotas estão tracejadas.

\subsection{Equação reduzida}

\subsubsection{Hipérbole com centro na origem e focos sobre o eixo $x$}

Pela Figura 4.3 temos os pontos $O(0,0), F_{1}(-c, 0), F_{2}(c, 0), A_{1}(-a, 0), A_{2}(a, 0)$ e $P(x, y)$.

Como $P \in$ a hipérbole temos pela definição que $\left|P F_{1}-P F_{2}=2 a\right|$, então segue da Figura 4.3 , que:

$$
\begin{gathered}
\sqrt{(x+c)^{2}+(y-0)^{2}}-\sqrt{(x-c)^{2}+(y-0)^{2}}= \pm 2 a \\
\sqrt{(x+c)^{2}+y^{2}}=\sqrt{(x-c)^{2}+y^{2}} \pm 2 a
\end{gathered}
$$

elevando ao quadrado membro a membro, temos:

$$
(x+c)^{2}+y^{2}=(x-c)^{2}+y^{2} \pm 4 a \sqrt{(x-c)^{2}+y^{2}}+4 a^{2}
$$




$$
\begin{aligned}
4 c x-4 a^{2} & = \pm 4 a \sqrt{(x-c)^{2}+y^{2}} \\
c x-a^{2} & = \pm a \sqrt{(x-c)^{2}+y^{2}}
\end{aligned}
$$

elevando novamente membro a membro ao quadrado, temos:

$$
\begin{gathered}
c^{2} x^{2}-2 a^{2} c x+a^{4}=a^{2} x^{2}-2 a^{2} c x+a^{2} c^{2}+a^{2} y^{2} \\
\left(c^{2}-a^{2}\right) x^{2}-a^{2} y^{2}=a^{2}\left(c^{2}-a^{2}\right)
\end{gathered}
$$

substituindo $\left(c^{2}-a^{2}\right)$ por $b^{2}$, pois $c^{2}=a^{2}+b^{2}$, temos:

$$
b^{2} x^{2}-a^{2} y^{2}=a^{2} b^{2}
$$

dividindo membro a membro por $a^{2} b^{2}$ teremos a equação

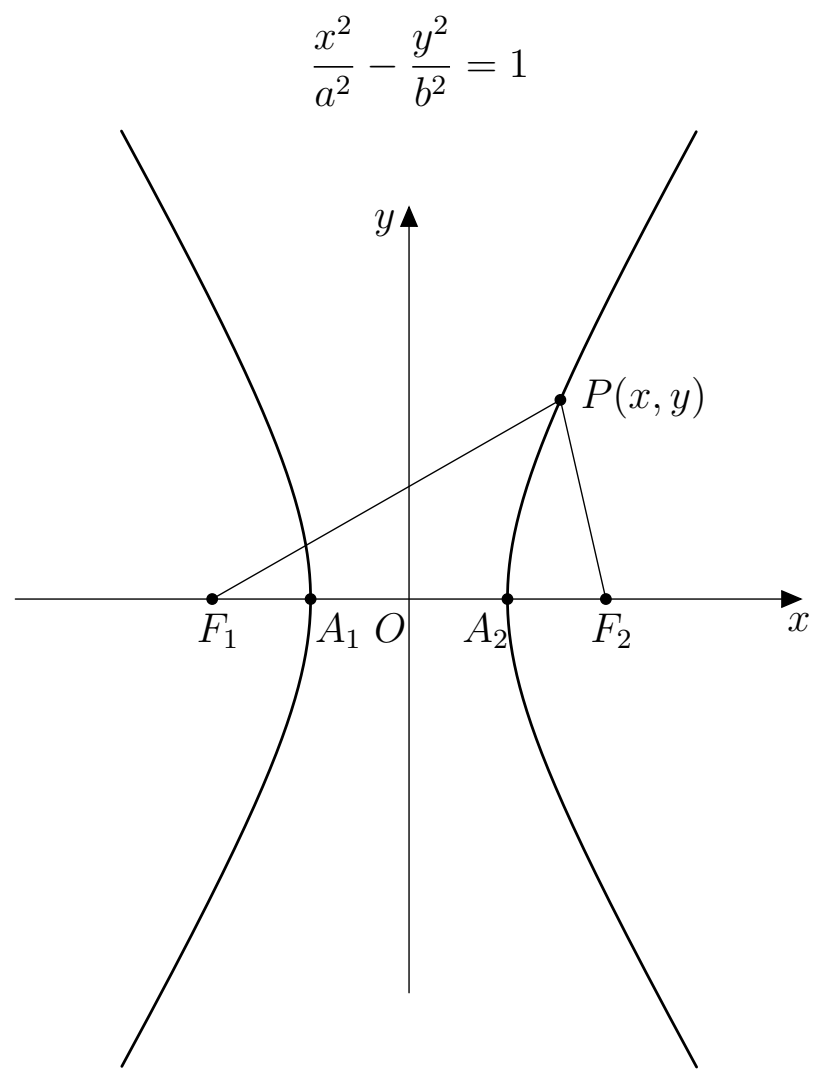

Figura 4.3: Hipérbole com centro na origem e focos sobre o eixo $x$

Assim, a equação

$$
\frac{x^{2}}{a^{2}}-\frac{y^{2}}{b^{2}}=1
$$

representa a hipérbole de foco nos pontos $( \pm c, 0) \operatorname{com} c=\sqrt{a^{2}+b^{2}}$. 


\subsubsection{Hipérbole com centro na origem e focos sobre o eixo $y$}

Pela Figura 4.4 temos os pontos $O(0,0), F_{1}(0, c), F_{2}(0, c), A_{1}(0,-a), A_{2}(0, a)$ e $P(x, y)$.

Como $P \in$ a hipérbole temos pela definição que $\left|P F_{1}-P F_{2}=2 a\right|$, então segue da Figura 4.4, que:

$$
\begin{gathered}
\sqrt{(x-0)^{2}+(y+c)^{2}}-\sqrt{(x-0)^{2}+(y-c)^{2}}= \pm 2 a \\
\sqrt{x^{2}+(y+c)^{2}}=\sqrt{x^{2}+(y-c)^{2}} \pm 2 a
\end{gathered}
$$

Elevando ao quadrado membro a membro, temos:

$$
\begin{gathered}
x^{2}+(y+c)^{2}=x^{2}+(y-c)^{2} \pm 4 a \sqrt{x^{2}+(y-c)^{2}}+4 a^{2} \\
4 c y-4 a^{2}= \pm 4 a \sqrt{x^{2}+(y-c)^{2}} \\
c y-a^{2}= \pm a \sqrt{x^{2}+(y-c)^{2}}
\end{gathered}
$$

elevando novamente membro a membro ao quadrado, temos:

$$
\begin{gathered}
c^{2} y^{2}-2 a^{2} c y+a^{4}=a^{2} x^{2}-2 a^{2} c y+a^{2} c^{2}+a^{2} y^{2} \\
\left(c^{2}-a^{2}\right) y^{2}-a^{2} x^{2}=a^{2}\left(c^{2}-a^{2}\right)
\end{gathered}
$$

substituindo $\left(c^{2}-a^{2}\right)$ por $b^{2}$, pois $c^{2}=a^{2}+b^{2}$, temos:

$$
b^{2} y^{2}-a^{2} x^{2}=a^{2} b^{2}
$$

dividindo membro a membro por $a^{2} b^{2}$ teremos a equação

$$
\frac{y^{2}}{a^{2}}-\frac{x^{2}}{b^{2}}=1
$$




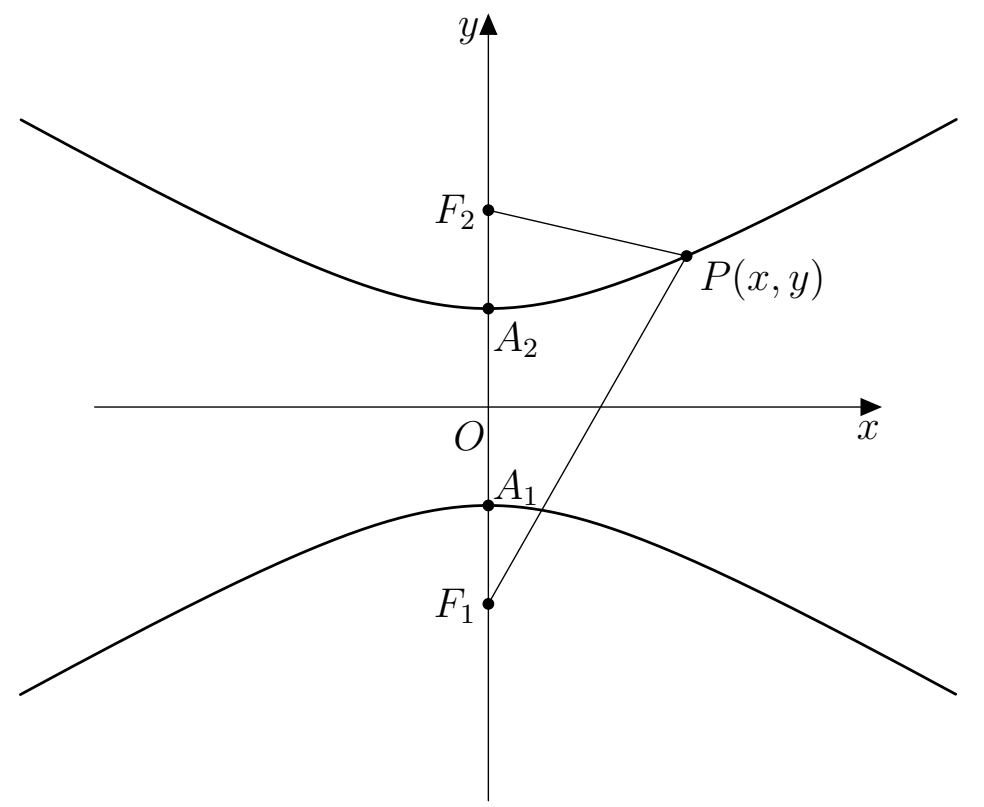

Figura 4.4: Hipérbole com centro na origem e focos sobre o eixo $y$

\subsubsection{Hipérbole com centro fora da origem e eixo real paralelo ao eixo $x$}

Pela Figura 4.5 temos os pontos $O\left(x_{0}, y_{0}\right), F_{1}\left(x_{0}-c, y_{0}\right), F_{2}\left(x_{0}+c, y_{0}\right)$, $A_{1}\left(x_{0}-a, y_{0}\right), A_{2}\left(x_{0}+a, y_{0}\right)$ e $P(x, y)$.

Como $P \in$ a hipérbole temos pela definição que $\left|P F_{1}-P F_{2}=2 a\right|$, então segue da Figura 4.5, que:

$$
\begin{aligned}
& \sqrt{\left[\left(x-x_{0}\right)+c\right]^{2}+\left(y-y_{0}\right)^{2}}-\sqrt{\left[\left(x-x_{0}\right)-c\right]^{2}+\left(y-y_{0}\right)^{2}}= \pm 2 a \\
& \sqrt{\left[\left(x-x_{0}\right)+c\right]^{2}+\left(y-y_{0}\right)^{2}}=\sqrt{\left[\left(x-x_{0}\right)-c\right]^{2}+\left(y-y_{0}\right)^{2}} \pm 2 a
\end{aligned}
$$

Elevando ao quadrado membro a membro, temos:

$$
\begin{gathered}
{\left[\left(x-x_{0}\right)+c\right]^{2}+\left(y-y_{0}\right)^{2}=\left[\left(x-x_{0}\right)-c\right]^{2}+\left(y-y_{0}\right)^{2} \pm 4 a \sqrt{\left[\left(x-x_{0}\right)-c\right]^{2}+\left(y-y_{0}\right)^{2}}+4 a^{2}} \\
4 c\left(x-x_{0}\right)-4 a^{2}= \pm 4 a \sqrt{\left[\left(x-x_{0}\right)-c\right]^{2}+\left(y-y_{0}\right)^{2}} \\
c\left(x-x_{0}\right)-a^{2}= \pm a \sqrt{\left[\left(x-x_{0}\right)-c\right]^{2}+\left(y-y_{0}\right)^{2}}
\end{gathered}
$$

elevando novamente membro a membro ao quadrado, temos:

$c^{2}\left(x-x_{0}\right)^{2}-2 a^{2} c\left(x-x_{0}\right)+a^{4}=a^{2}\left(x-x_{0}\right)^{2}-2 a^{2} c\left(x-x_{0}\right)+a^{2} c^{2}+a^{2}\left(y-y_{0}\right)^{2}$ 


$$
\left(c^{2}-a^{2}\right)\left(x-x_{0}\right)^{2}-a^{2}\left(y-y_{0}\right)^{2}=a^{2}\left(c^{2}-a^{2}\right)
$$

substituindo $\left(c^{2}-a^{2}\right)$ por $b^{2}$, pois $c^{2}=a^{2}+b^{2}$, temos:

$$
b^{2}\left(x-x_{0}\right)^{2}-a^{2}\left(y-y_{0}\right)^{2}=a^{2} b^{2}
$$

dividindo membro a membro por $a^{2} b^{2}$ teremos a equação

$$
\frac{\left(x-x_{0}\right)^{2}}{a^{2}}-\frac{\left(y-y_{0}\right)^{2}}{b^{2}}=1
$$

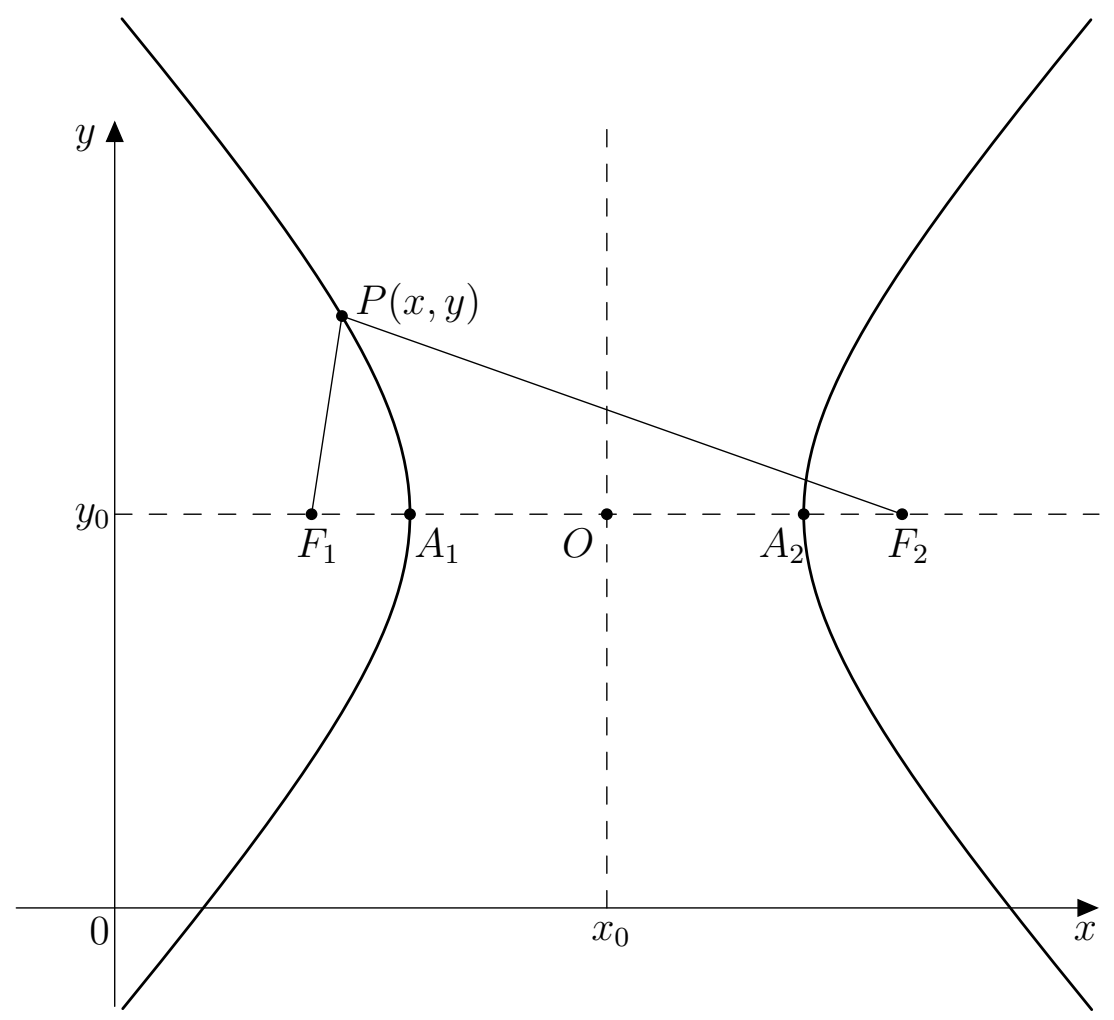

Figura 4.5: Hipérbole com centro fora da origem e eixo real paralelo ao eixo $x$

\subsubsection{Hipérbole com centro fora da origem e eixo real paralelo ao eixo $y$}

Pela Figura 4.6 temos os pontos $O\left(x_{0}, y_{0}\right), F_{1}\left(x_{0}, y_{0}-c\right), F_{2}\left(x_{0}, y_{0}+c\right)$, $A_{1}\left(x_{0}, y_{0}-a\right), A_{2}\left(x_{0}, y_{0}+a\right)$ e $P(x, y)$. 
Como $P \in$ a hipérbole temos pela definição que $\left|P F_{1}-P F_{2}=2 a\right|$, então segue da Figura 4.6, que:

$$
\begin{gathered}
\sqrt{\left(x-x_{0}\right)^{2}+\left[\left(y-y_{0}\right)+c\right]^{2}}-\sqrt{\left(x-x_{0}\right)^{2}+\left[\left(y-y_{0}\right)-c\right]^{2}}= \pm 2 a \\
\sqrt{\left(x-x_{0}\right)^{2}+\left[\left(y-y_{0}\right)+c\right]^{2}}=\sqrt{\left(x-x_{0}\right)^{2}+\left[\left(y-y_{0}\right)-c\right]^{2}} \pm 2 a
\end{gathered}
$$

Elevando ao quadrado membro a membro, temos:

$$
\begin{gathered}
\left(x-x_{0}\right)^{2}+\left[\left(y-y_{0}\right)+c\right]^{2}=\left(x-x_{0}\right)^{2}+\left[\left(y-y_{0}\right)-c\right]^{2} \pm 4 a \sqrt{\left(x-x_{0}\right)^{2}+\left[\left(y-y_{0}\right)-c\right]^{2}}+4 a^{2} \\
4 c\left(y-y_{0}\right)-4 a^{2}= \pm 4 a \sqrt{\left(x-x_{0}\right)^{2}+\left[\left(y-y_{0}\right)-c\right]^{2}} \\
c\left(y-y_{0}\right)-a^{2}= \pm a \sqrt{\left(x-x_{0}\right)^{2}+\left[\left(y-y_{0}\right)-c\right]^{2}}
\end{gathered}
$$

elevando novamente membro a membro ao quadrado, temos:

$$
\begin{gathered}
c^{2}\left(y-y_{0}\right)^{2}-2 a^{2} c\left(y-y_{0}\right)+a^{4}=a^{2}\left(x-x_{0}\right)^{2}-2 a^{2} c\left(y-y_{0}\right)+a^{2} c^{2}+a^{2}\left(y-y_{0}\right)^{2} \\
\left(c^{2}-a^{2}\right)\left(y-y_{0}\right)^{2}-a^{2}\left(x-x_{0}\right)^{2}=a^{2}\left(c^{2}-a^{2}\right)
\end{gathered}
$$

substituindo $\left(c^{2}-a^{2}\right)$ por $b^{2}$, pois $c^{2}=a^{2}+b^{2}$, temos:

$$
b^{2}\left(y-y_{0}\right)^{2}-a^{2}\left(x-x_{0}\right)^{2}=a^{2} b^{2}
$$

dividindo membro a membro por $a^{2} b^{2}$ teremos a equação

$$
\frac{\left(y-y_{0}\right)^{2}}{a^{2}}-\frac{\left(x-x_{0}\right)^{2}}{b^{2}}=1
$$




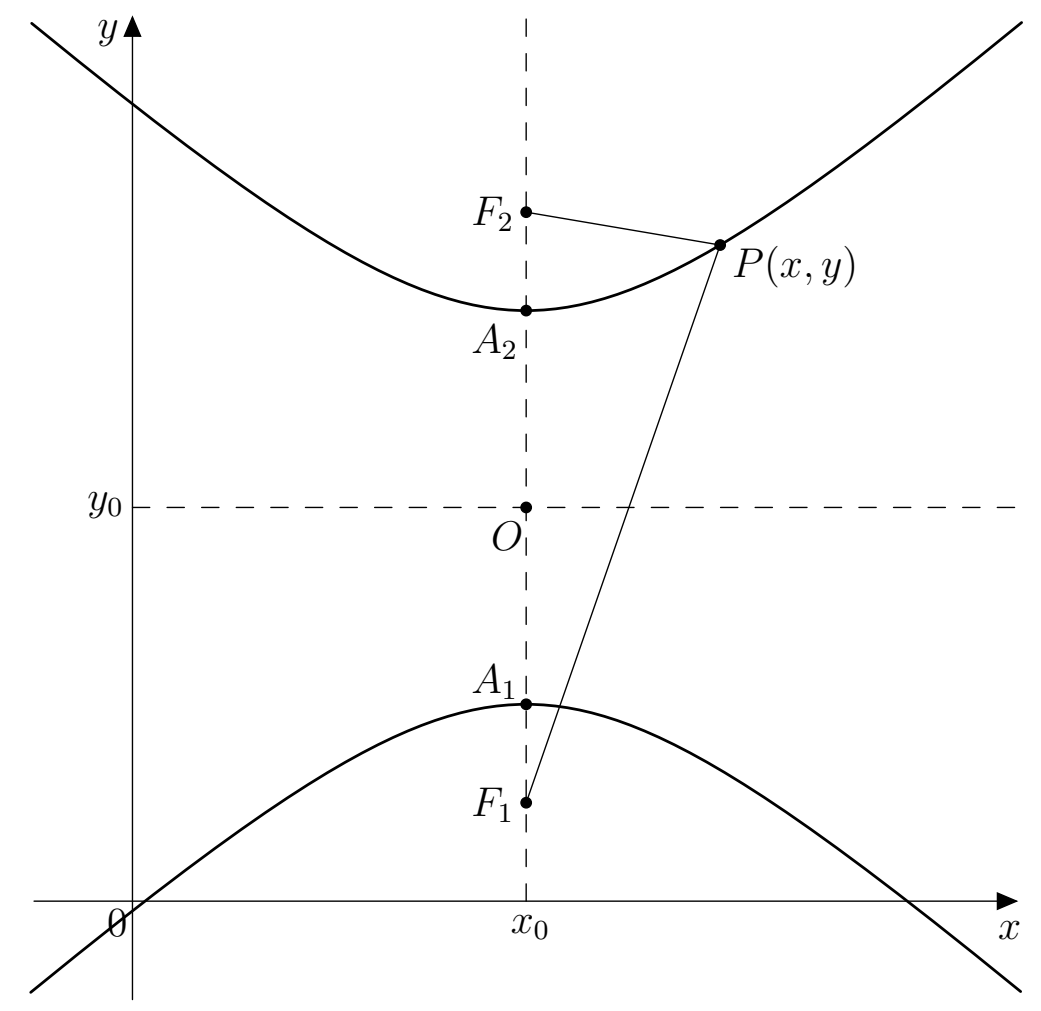

Figura 4.6: Hipérbole com centro fora da origem e eixo real paralelo ao eixo $y$ 


\section{Capítulo 5}

\section{Equação geral das cônicas}

Nesse capítulo vamos analisar uma cônica por sua equação geral. Esse tema é abordado em qualquer bom texto de geometria analítica, dessa forma utilizaremos um procedimento apenas em linhas gerais.

Para nós, uma cônica é uma forma geométrica obtida da seção de um cone de folha dupla cortado por um plano que não passa pelo vértice do cone (a fim de evitar os casos degenerados), ou seja, temos a elipse, a circunferência, a hipérbole e a parábola. Segundo Boulos [3], uma cônica é o conjunto dos pontos que satisfazem uma equação do segundo grau em duas variáveis da forma

$$
a x^{2}+b x y+c y^{2}+d x+e y+f=0
$$

em relação ao eixo ortogonal de coordenadas, onde $a x^{2}, b x y$ e $c x^{2}$ são chamados de termos quadráticos, bxy o termo quadrático misto, dx e ey são os termos lineares e $f$ é o termo independente. Faz assim para facilitar sua análise.

Note que nem sempre as soluções de (5.1) são cônicas. Os outros casos serão chamados de cônicas degeneradas, podendo ser um ponto, uma reta, retas paralelas, retas concorrentes ou até um conjunto vazio, como veremos nos exemplos a seguir:

- Conjunto vazio: a equação $x^{2}+y^{2}+10=0$ não é satisfeita por nenhum $(x, y)$.

- Conjunto formado por um ponto: $(x-1)^{2}+(y-1)^{2}=0$, ou seja, $x^{2}+y^{2}-2 x-2 y+2=0$, admite apenas a solução $(1,1)$. 
- Reta: a equação $(x-y)^{2}=0$, isto é, $x^{2}-2 x y+y^{2}=0$, descreve a reta $r: x=y$.

- Retas paralelas: $(x+y+1)(x+y-1)=0$, ou seja, $x^{2}+2 x y+y^{2}-1=0$, descreve as retas $r: x+y+1=0$ e $s: x+y-1=0$.

- Retas concorrentes: $(x+y)(x-y)=0$, isto é, $x^{2}-y^{2}=0$, descreve as retas $r: x=-y$ e $s: x=y$.

\subsection{Eliminando o termo misto}

As equações reduzidas das cônicas não apresentam o termo misto. Podemos eliminá-lo da equação geral por meio de um rotação de eixos, ficando com a equação geral da forma $a x^{2}+b y^{2}+c x+d y+e=0$. Para isso, utilizaremos as fórmulas de rotação que estabelecem as relações entre as coordenadas $(x, y)$ de um ponto $P$, em relação ao sistema $x y$, com suas coordenadas $(\tilde{x}, \tilde{y})$ em relação ao sistema $\tilde{x} \tilde{y}$. Veja a Figura 5.1.

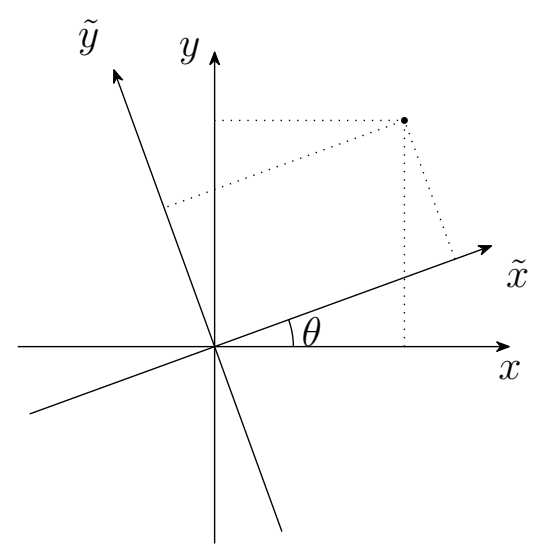

Figura 5.1: O sistema $\tilde{x}, \tilde{y}$ é obtido pela rotação de ângulo $\theta$ no sentido anti-horário.

As coordenadas de $P$ no sistema $x, y$ são obtidas das coordenadas no sistema $\tilde{x}, \tilde{y}$ pela rotação de ângulo $\theta$ no sentido anti-horário. As relações são

$$
\begin{aligned}
& x=\tilde{x} \cos (\theta)-\tilde{y} \operatorname{sen}(\theta), \\
& y=\tilde{x} \operatorname{sen}(\theta)+\tilde{y} \cos (\theta) .
\end{aligned}
$$


Aplicando as fórmulas (5.2)-(5.3) à equação geral de uma cônica obtemos sua equação em relação ao sistema de eixos $\tilde{x} \tilde{y}$ :

$$
\tilde{a} \tilde{x}^{2}+\tilde{b} \tilde{x} \tilde{y}+\tilde{c} \tilde{y}^{2}+\tilde{d} \tilde{x}+\tilde{e} \tilde{y}+\tilde{f}=0
$$

com

$$
\tilde{b}=b \cos (2 \theta)-(a-c) \operatorname{sen}(2 \theta) .
$$

Fazendo $\tilde{b}=0$ acima, obtemos

$$
b \cos (2 \theta)=(a-c) \operatorname{sen}(2 \theta) .
$$

Se $a \neq c$, então calculamos

$$
\tan (2 \theta)=\frac{b}{a-c}
$$

e podemos tomar $\theta=\frac{1}{2} \arctan \left(\frac{b}{a-c}\right)$. Se $a=c, 5.4$ está satisfeita se $\theta=45^{\circ}$.

Para deixar mais leve a leitura, após aplicarmos essa mudança de coordenadas, continuaremos a utilizar as letras $a, c \ldots, x, y$ em vez de $\tilde{a}, \tilde{c}, \ldots, \tilde{x}, \tilde{y}$. Assim, 5.1 pode sempre ser colocada na forma sem o termo misto

$$
a x^{2}+c y^{2}+d x+e y+f=0 .
$$

Cabe observar que a rotação é uma isometria, isto é, uma transformação que mantém distâncias, preservando a forma dos conjuntos.

\subsection{Eliminando os termos lineares}

Se for necessário eliminar termos lineares, quando $a \neq 0$ ou $c \neq 0$, podemos fazê-lo por completamento de quadrado, o que nos dá a translação que reduz a equação à equação reduzida de alguma cônica, vista nos capítulos anteriores, ou numa equação de cônica degenerada. A partir daí, fica fácil classificarmos a cônica em questão.

\subsection{Exemplos}

Exemplo 5.1. O gráfico da função $y=\frac{1}{x}$. 
Podemos reescrever a equação do gráfico como

$$
x y-1=0 .
$$

Como $a=c=0$, aplicamos a rotação de $45^{\circ}$, ou seja,

$$
x=\frac{\sqrt{2}}{2}(\tilde{x}-\tilde{y}), \quad y=\frac{\sqrt{2}}{2}(\tilde{x}+\tilde{y}) .
$$

Aplicando (5.7) em (5.6), ficamos com

$$
\frac{1}{2}(\tilde{x}-\tilde{y})(\tilde{x}+\tilde{y})-1=0 \Longrightarrow \frac{\tilde{x}^{2}}{2}-\frac{\tilde{y}^{2}}{2}=1,
$$

que é a equação reduzida da hipérbole de focos $(\tilde{x}, \tilde{y})=( \pm 2 \sqrt{2}, 0)$. Retornando às variáveis $x, y$, mostramos que o gráfico da função $y=\frac{1}{x}$ é uma hipérbole com focos $(2,2)$ e $(-2,-2)$.

Exemplo 5.2. Equação $16 x^{2}+25 y^{2}-96 x-200 y-144=0$.

Não será necessário aplicar a rotação, pois a equação não apresenta termo misto. Então para encontrarmos a equação reduzida, vamos completar quadrados.

$$
\begin{gathered}
16 x^{2}+25 y^{2}-96 x-200 y-144=0 \\
16 x^{2}-96 x+144+25 y^{2}-200 y+400-400=0 \\
16(x-3)^{2}+25(y-4)^{2}=400 \\
\frac{(x-3)^{2}}{25}+\frac{(y-4)^{2}}{16}=1,
\end{gathered}
$$

que é a equação reduzida da elipse de centro $(3,4)$, eixo maior 5 e eixo menor 4.

Exemplo 5.3. Equação $y^{2}+2 x-6 y+1=0$ Vamos aplicar a translação completando quadrado.

$$
\begin{gathered}
y^{2}+2 x-6 y+1=0 \\
y^{2}-6 y+9-9+2 x+1=0 \\
(y-3)^{2}=-2(x-4),
\end{gathered}
$$

que é a equação reduzida da parábola com vértice em $(4,3)$. 


\section{Capítulo 6}

\section{As esferas de Dandelin}

Nesse capítulo demonstraremos como Germinal Dandelin (1794-1847) e Adolphe Quetelet (1796-1874) mostraram que a elipse, a hipérbole e a parábola podem ser obtidas seccionando-se um cone duplo por meio de um plano de inclinação variável em relação ao seu eixo.

Seguiremos como Gilberto Geraldo Garbi em "A Rainha das Ciências" [6].

\subsection{Elipse}

Sejam, como na Figura 6.1, uma das folhas de um cone duplo reto, $\alpha$ um plano que intercepta esse cone em todas as suas geratrizes e as esferas $E_{1} \mathrm{e}$ $E_{2}$, que se apresentam em semiespaços distintos em relação a $\alpha$, inscritas no cone e tangentes a $\alpha$.

Sejam $F_{1}$ e $F_{2}$, respectivamente, os pontos de tangência $E_{1}$ e $E_{2}$ com o plano $\alpha$ e $C_{1}$ e $C_{2}$ as circunferências geradas pelas interseções de $E_{1}$ e $E_{2}$ com a superfície do cone.

Tomando um ponto $P$ qualquer da interseção do cone com o plano $\alpha$ e ligando $P$ ao vértice $V$ do cone, teremos a reta $P V$ se intersectando com $C_{1}$ e $C_{2}$ nos pontos $T_{1}$ e $T_{2}$, tangentes às esferas $E_{1}$ e $E_{2}$ respectivamente.

Os segmentos $P T_{1}$ e $P F_{1}$ são tangentes à esfera $E_{1}$ e, por potência de pontd ${ }^{1}$ eles são congruentes. Pelo mesmo motivo, $P T_{2}$ e $P F_{2}$ também são congruentes.

\footnotetext{
${ }^{1} \mathrm{O}$ plano $\beta$ que contém os pontos $P, T_{1}$ e $F_{1}$ intersecta a esfera $E_{1}$ numa circunferência $\lambda$ que tem segmentos tangentes $P T_{1}$ e $P F_{1}$, congruentes pela potência do ponto $P$ em relação a $\lambda$.
} 
Logo, temos:

$$
P T_{1}=P F_{1}, \quad P T_{2}=P F_{2} \Longrightarrow P F_{1}+P F_{2}=P T_{1}+P T_{2} .
$$

Como $P T_{1}+P T_{2}$ é constante para qualquer $P$ pertencente à interseção do cone com o plano $\alpha$, pois as duas circunferências $C_{1}$ e $C_{2}$ estão contidas em planos paralelos, tal interseção do plano $\alpha$ com o cone é uma elipse.

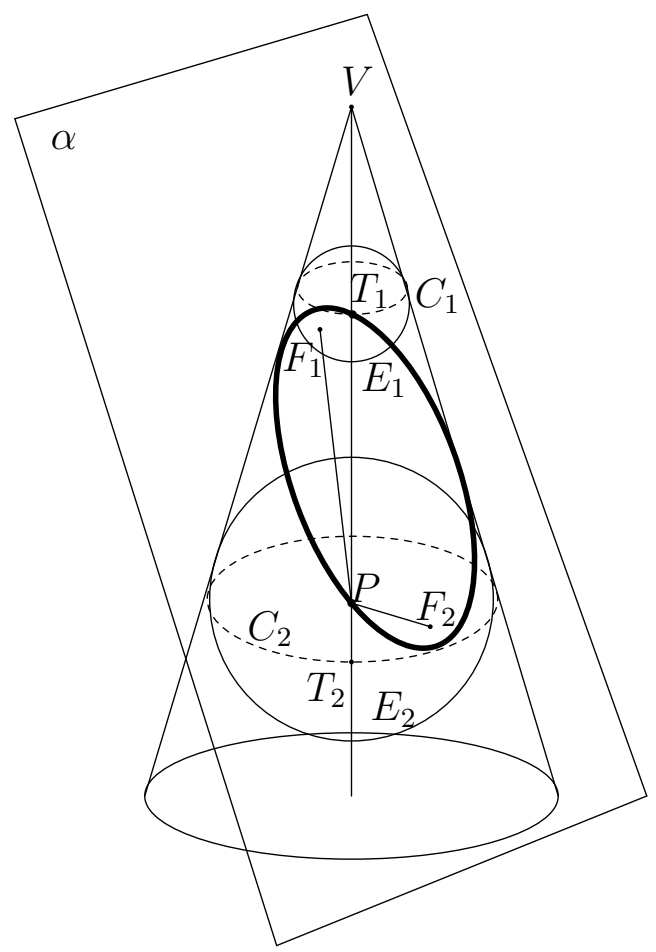

Figura 6.1: Esferas de Dandelin da elipse.

\subsection{Hipérbole}

Sejam, como na Figura 6.2, um cone duplo reto, um plano a seccionando suas duas folhas e as esferas $E_{1}$ e $E_{2}$ inscritas uma em cada folha do cone e tangentes ao plano. Sejam $C_{1}$ e $C_{2}$ as circunferências geradas pelas interseções de $E_{1}$ e $E_{2}$ com superfície do cone e $F_{1}$ e $F_{2}$ os pontos de tangência das esferas com o plano. 
Tomando um ponto $P$ qualquer pertencente à interseção do plano $\alpha$ com o cone e ligando $P$ ao vértice $V$ do cone, teremos a reta $P V$ intersectando as circunferências $C_{1}$ e $C_{2}$ nos pontos $T_{1}$ e $T_{2}$, tangentes às esferas $E_{1}$ e $E_{2}$, respectivamente.

Os segmentos $P T_{1}$ e $P F_{1}$ são tangentes à esfera $E_{1}$ e por potência de ponto (ver nota de rodapé na página 41), eles são congruentes, pelo mesmo motivo, $P T_{2}$ e $P F_{2}$ também são congruentes.

Logo,

$$
P T_{1}=P F_{1}, \quad P T_{2}=P F_{2} \Longrightarrow\left|P T_{1}-P T_{2}\right|=\left|P F_{1}-P F_{2}\right|=T_{1} T_{2}
$$

Como $T_{1} T_{2}=V T_{1}+V T_{2}$ que é constante, pois as circunferências $C_{1}$ e $C_{2}$ estão contidas em planos paralelos, temos $\left|P F_{1}-P F_{2}\right|$ constante para qualquer $P$ pertencente à interseção do plano $\alpha$ com cone, assim tal interseção é uma hipérbole.

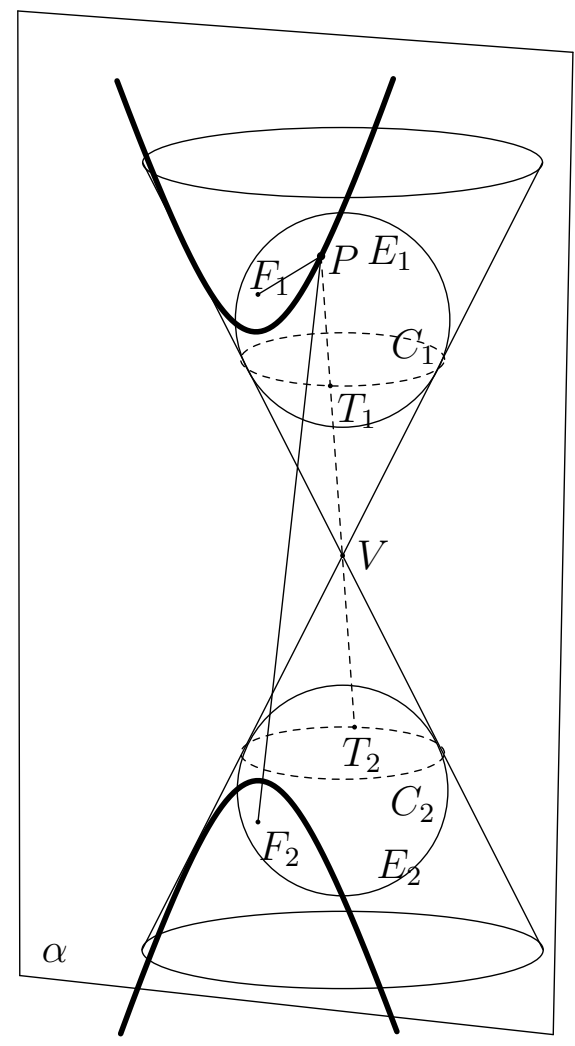

Figura 6.2: Esferas de Dandelin da hipérbole. 


\subsection{Parábola}

Vamos agora ao caso em que o plano $\alpha$ secciona o cone paralelamente a uma e somente uma das geratrizes $g$ de um cone duplo reto.

Sejam, como na Figura 6.3. uma das folhas de uma superfície cônica circular e sobre ela uma geratriz qualquer $g$. Sendo $E$ uma esfera inscrita em um cone e $\alpha$ um plano que secciona esse cone, tangenciando a esfera no ponto $F$, de modo que esse plano seja paralelo a uma e somente geratriz desse cone, a saber $g$. Seja $C_{1}$ a circunferência gerada pela interseção da esfera $E$ com o cone, e tome um plano $\gamma$ que contém a circunferência $C_{1}$, onde a interseção de $\alpha$ com $\gamma$ é uma reta $r_{1}$. Na interseção de $\alpha$ com o cone, considere um ponto $P$ qualquer e por esse ponto tome um plano $\beta$ paralelo ao plano $\gamma$.

Seja $C_{2}$ a circunferência formada pela interseção do plano $\beta$ com o cone e seja $r_{2}$ a interseção do plano $\alpha$ com o plano $\beta$, sendo assim, temos a reta $r_{1}$ paralela à reta $r_{2}$.

Imaginando um plano tangente à superfície do cone e que contenha a geratriz $g$, teremos esse plano paralelo ao plano $\alpha$ e assim a interseção desse plano com $\beta$ será uma reta $s$ paralela à reta $r_{2}$ e tangente à circunferência $C_{2}$ no ponto $S$.

Traçando um segmento $P Q$ perpendicular a $r_{1}$ e $r_{2}$ e por $F$ um segmento $R G$ que também é perpendicular a $r_{1}$ e $r_{2}$, teremos $P Q=R G$. A geratriz $g$ e a reta do segmento $R G$ são paralelas por serem, respectivamente, perpendiculares às paralelas $s$ e $r_{2}$, de forma que, sendo $I \in C_{1} \cap g, I S=R G$. Ligando $P$ a $V$, seja $T$ a interseção de $V P$ com a circunferência $C_{1}$. Como $I S$ e $P T$ são geratrizes do tronco de cone formado por planos paralelos, temos $I S=P T$ e por potência de ponto na esfera $E$ temos $P T=P F, \log$ o

$$
P Q=G R=S I=P T=P F
$$

então

$$
P Q=P F
$$

Portanto, a curva formada pela interseção do plano $\alpha$ com o cone é a parábola com diretriz $r_{1}$ e foco $F$. 


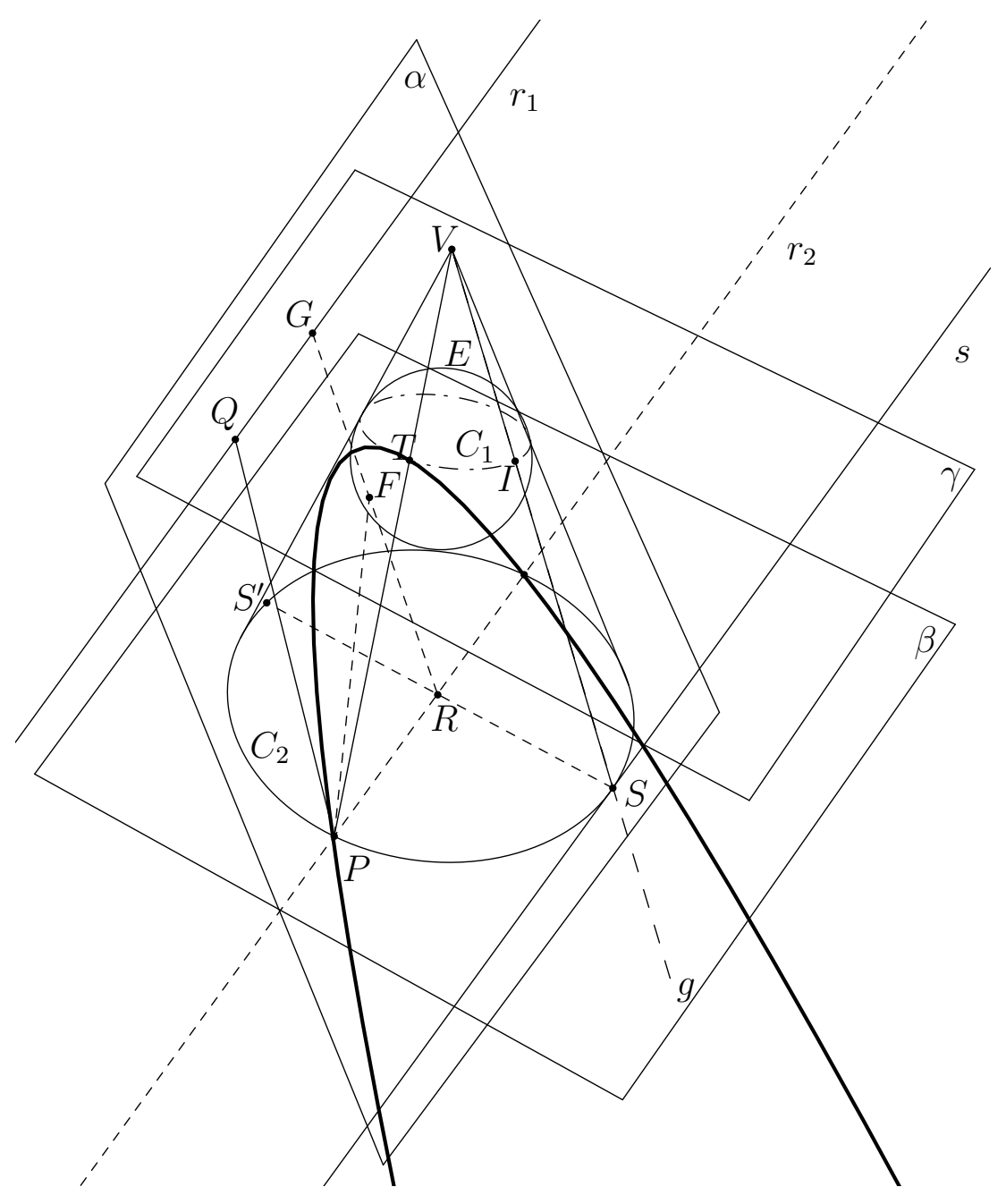

Figura 6.3: Esfera de Dandelin da parábola. 


\section{Capítulo 7}

\section{Aplicações das cônicas}

Daremos aqui algumas das aplicações mais imediatas e úteis das cônicas. Suas propriedades geométricas permitem deduzir propriedades notáveis de reflexão em sua superfície. Aqui estaremos pensando em superfícies de revolução geradas pela rotação das cônicas em torno de seus eixos de simetria, e estudaremos os espelhos obtidos por estas superfícies.

Ao final veremos também o sistema de navegação marítima LORAN que apesar de não ter relação com as propriedades reflexivas das cônicas, apresenta uma aplicação muito importante da hipérbole.

\subsection{Espelhos parabólicos}

Os sinais captados pela antena parabólica podem ser considerados todos paralelos por conta da grande distância que percorrem para chegar à superfície do planeta. As antenas parabólicas fazem com que esses sinais recebidos sejam concentrados em um único ponto, que é o foco da parábola, onde se encontra o receptor dos sinais de TV.

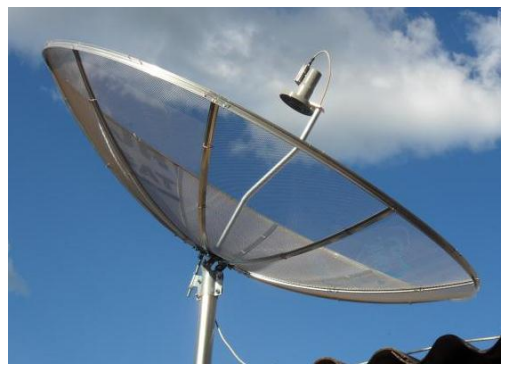


Com dois espelhos parabólicos, podemos construir um espelho acústico, como o espelho do CDCC de São Carlos. As ondas sonoras emitidas por uma pessoa em um foco de uma das parábolas são refletidas paralelamente ao eixo de simetria comum, sendo captadas pelo outro espelho, que as concentra em seu foco, onde outra pessoa pode ouvir perfeitamente o que a primeira sussurra.

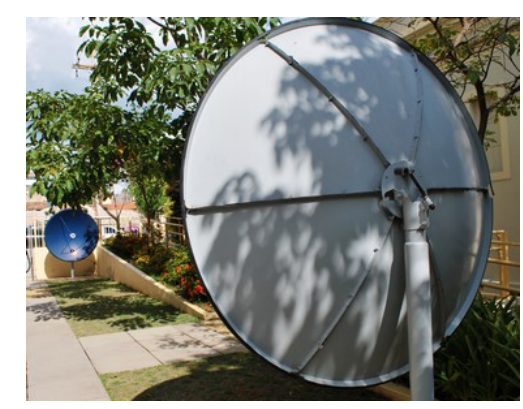

Agora vamos mostrar esse fato, usando uma adaptação das ideias de [20]. Consideremos um ponto $P$ da parábola de foco $F$ e diretriz $d$, e a reta $t$, bissetriz do ângulo $F \hat{P} D$. Vamos mostrar geometricamente que $t$ é tangente à parábola. Veja a Figura 7.1 .

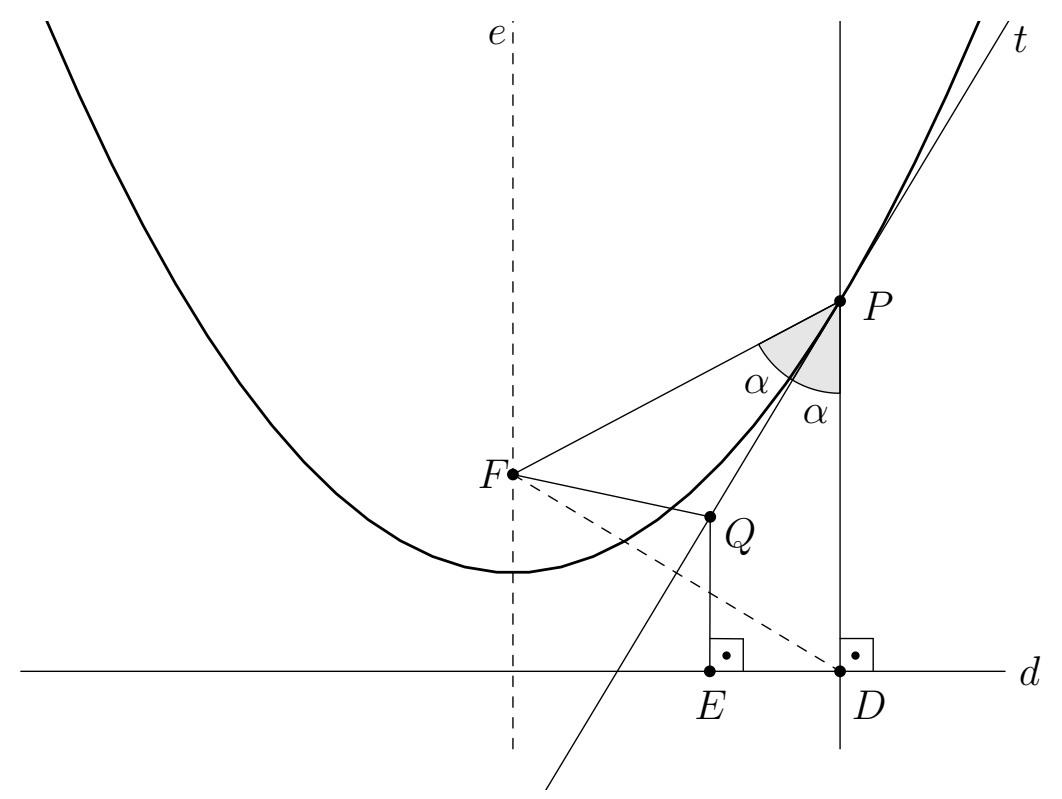

Figura 7.1: Reta tangente à parábola. 
No triângulo $\triangle P F D$, como $P F=P D$, a reta $t$, bissetriz do ângulo $F \hat{P} D$, é também mediana e altura. Em outras palavras, a reta $t$ é mediatriz do segmento $F D$. Seja agora $Q$, um ponto qualquer da reta $t$, distinto de $P$. Se $E$ é a projeção de $Q$ sobre $d$, temos:

$$
Q F=Q D>Q E
$$

Portanto, $Q$ é exterior à parábola. Ora, o ponto $P$ da reta $t$ pertence à parábola e todos os outro ponto de $t$ são exteriores. Logo, $t$ é tangente à parábola em $P$.

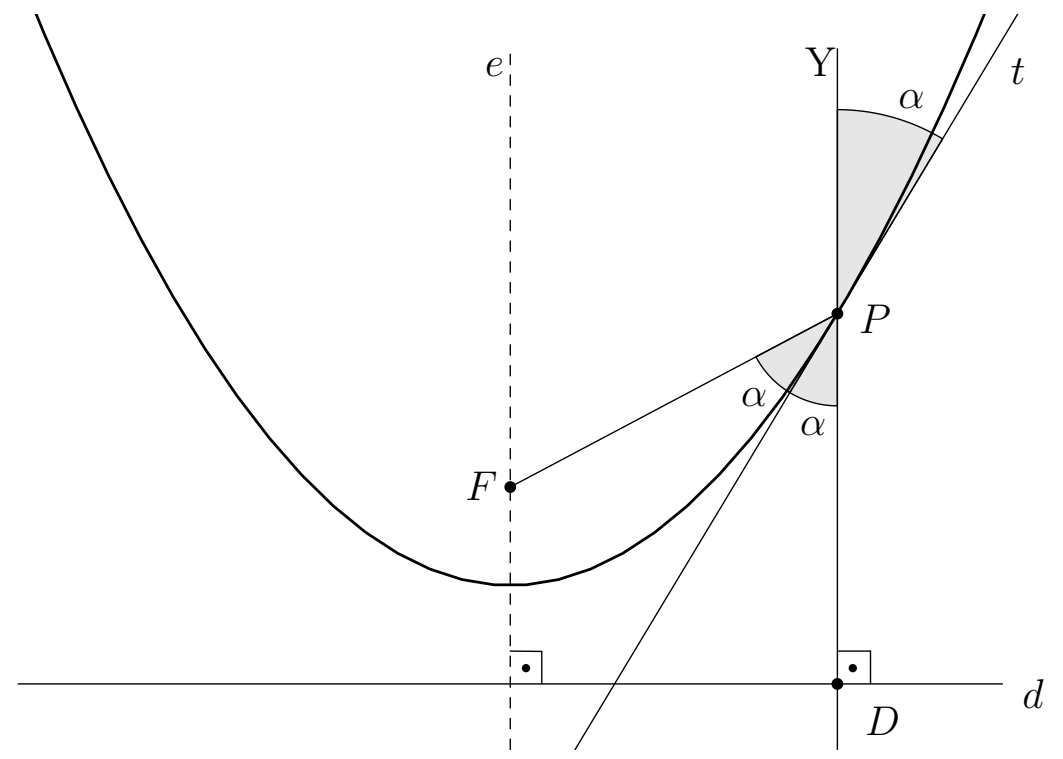

Figura 7.2: Reta tangente a parábola

Observe, na Figura 7.2, a semirreta $P Y$, prolongamento do segmento $D P$. Como a tangente à parábola em $P$ é bissetriz do ângulo $F \hat{P} D$, temos que $P Y$ e $P F$ fazem ângulos congruentes com essa tangente. Por isso, todo sinal recebido na direção do eixo da parábola toma a direção do foco após a reflexão.

\subsection{Espelhos elípticos}

As luminárias odontológicas de cabeça são feitas de refletores elípticos que focam a luz em um ponto específico da arcada dentária, de modo a não ofuscar a visão do paciente. 

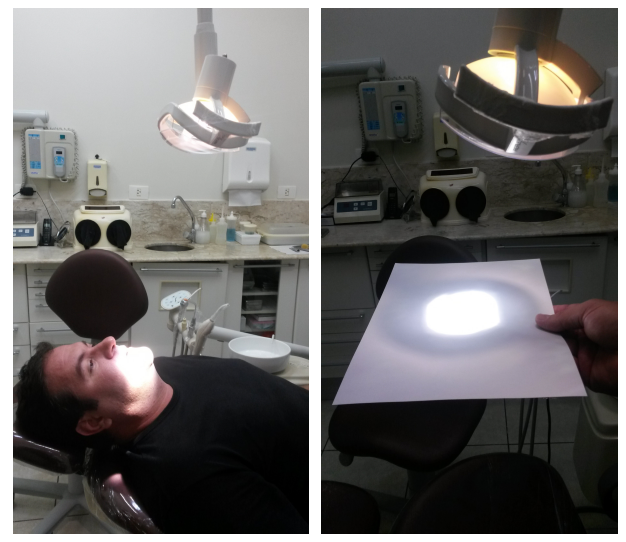

Um outro aparelho interessante, que se utiliza da elipse, é o da prática da Litotripsia, tratamento que é feito para eliminar pedras nos rins, por meio de um refletor elíptico, de modo a não danificar os tecidos e órgãos vizinhos. Desse modo o paciente não passa por cirurgia e sua recuperação é muito rápida. O refletor elíptico emite ondas sonoras de alta intensidade em um dos focos da elipse e a pedra fica no outro foco.

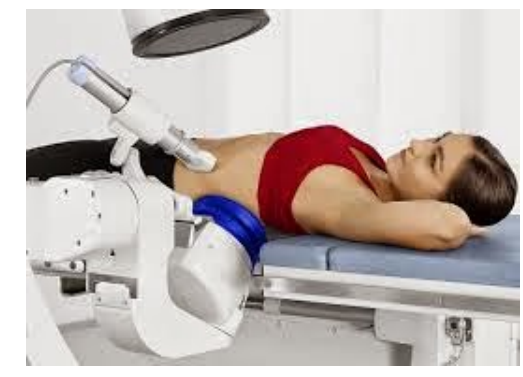

A mesa de bilhar elíptica é um interessante experimento que mostra na prática o que acontece nos dois equipamentos anteriores, onde a bola que sai de um foco, ao bater na "tabela", sofre reflexão cai na caçapa que se apresenta no outro foco da elipse.

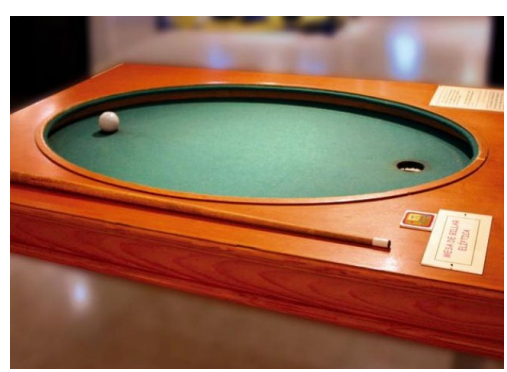


As galerias de sussurros são uma divertida aplicação de uma superfície elíptica. O teto da sala tem forma de elipsoide com focos à altura de uma pessoa marcados no chão. Duas pessoas nesses pontos podem conversar sussurrando e escutam-se perfeitamente, enquanto que as outras não as escutam. Há galerias de sussurros como, por exemplo, na Sala de Estátuas do Capitólio estadunidense.

A demonstração dessa propriedade de reflexão dos espelhos elípticos é dada a seguir, baseada em 18.

Seja $P$ um ponto na elipse $E$ e tomemos a reta $r$ bissetriz de um dos ângulos formados pelas retas $F_{1} P$ e $F_{2} P$ (o externo ao triângulo $\triangle F_{1} P F_{2}$ ) de tal forma que o ângulo entre entre $F_{1} P$ e $r$ seja igual ao ângulo entre $F_{2} P$ e $r$. Queremos mostrar que $r$ é tangente à elipse.

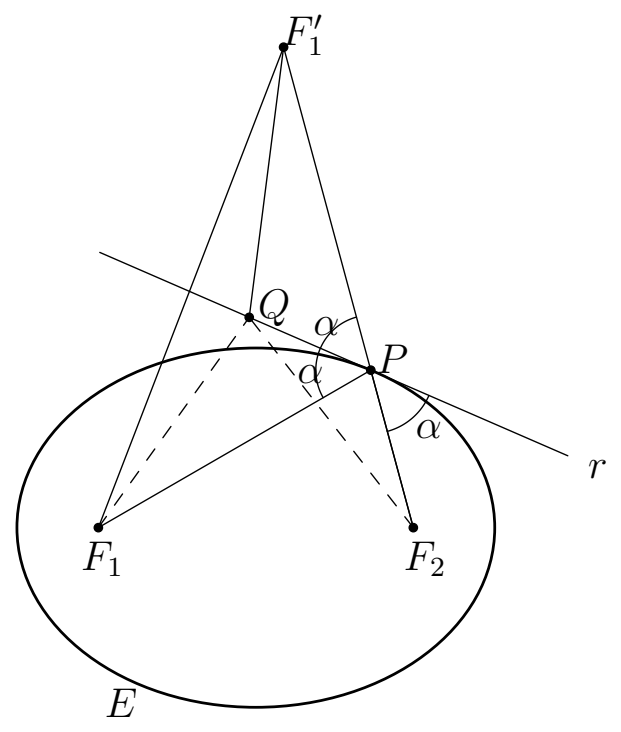

Figura 7.3: Reta tangente à elipse.

Seja $k=P F_{1}+P F_{2}$, a constante que determina a elipse, junto com seus focos. Tomemos sobre $r$ um ponto $Q \neq P$ e consideremos o ponto $F_{1}^{\prime}$, simétrico de $F_{1}$ em relação a $r$. A reta $r$ é então mediatriz de $F_{1} F_{1}^{\prime}$. Logo, $P F_{1}=P F_{1}^{\prime}$ e também $Q F_{1}=Q F_{1}^{\prime}$. Por construção, a reta $r$ faz ângulos congruentes com $P F_{1}$ e $P F_{2}$ e, pela simetria, os ângulos $Q \hat{P} F_{1}$ e $Q \hat{P} F_{1}^{\prime}$ são também congruentes. Daí, os segmentos $P F_{2}$ e $P F_{1}^{\prime}$ fazem ângulos congruentes com $r$ e, portanto, os pontos $F_{1}^{\prime}, P$ e $F_{2}$ são colineares. Então,

$$
k=P F_{1}+P F_{2}=P F_{1}^{\prime}+P F_{2}=F 1^{\prime} F_{2}<Q F_{1}^{\prime}+Q F_{2}=Q F_{1}+Q F_{2},
$$


onde a desigualdade acima vem da condição de existência do triângulo $\triangle F_{1}^{\prime} Q F_{2}$. Como $Q F_{1}+Q F_{2}>k$, concluímos que $P$ é o único ponto de $r$ que pertence à elipse, o que mostra que essa reta é tangente em $P$ a essa elipse. Um raio que parte de um foco a $P$ incide na reta $r$ tangente à elipse com ângulo $\alpha \mathrm{e}$ deve refletir com mesmo ângulo, passando consequentemente pelo outro foco. Veja a Figura 7.3 .

\subsection{Espelhos hiperbólicos}

Os espelhos hiperbólicos são amplamente utilizados na construção de telescópios refletores. Esses telescópios são uma evolução proposta pelo astrônomo francês Cassegrain dos telescópios produzidos por Isaac Newton, que resolveu o problema da imagem que era refletida dentro do tubo do telescópio, colocando um espelho plano e refletindo a imagem para o observador fora do tubo. No entanto, apesar de funcionar perfeitamente, esse espelho plano prejudicava a captação de luz, por ter um tamanho relativamente grande para as condições, enquanto que a proposta de Cassegrain era de utilizar um espelho hiperbólico que, como vamos mostrar, possui uma maior flexibilidade de movimentos, se ajustando melhor às necessidades do observador.

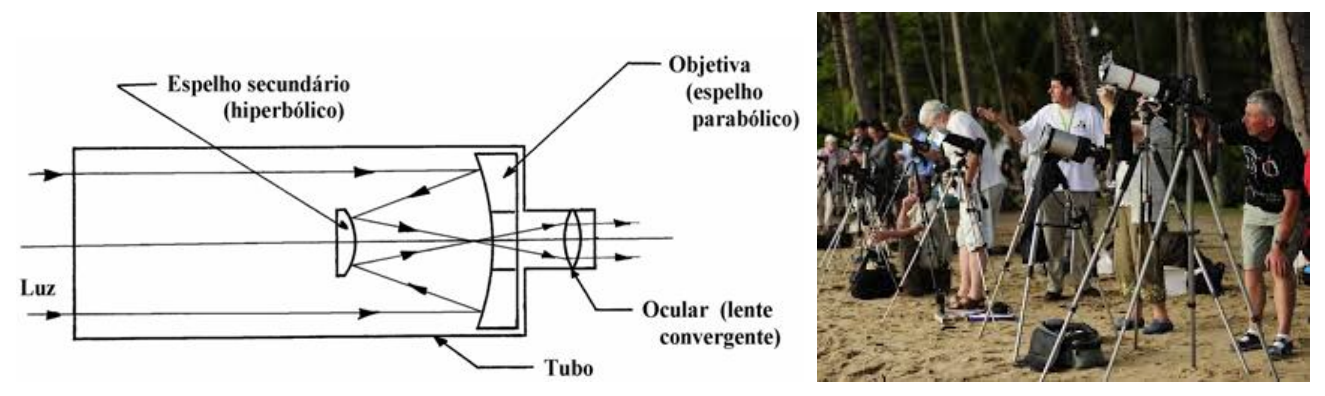

Agora vamos mostrar a propriedade de reflexão na hipérbole de um raio incidindo na direção de um ponto focal.

Seja $H$ uma folha de hipérbole com focos $F_{1}$ e $F_{2}$, mais próxima a $F_{2}$. Seja $P$ um ponto de $H$ e a constante $k=P F_{1}-P F_{2}$ que determina a hipérbole. A hipérbole divide o plano nas regiões $R_{1}: Q F_{1}-Q F_{2}<k$ e $R_{2}: Q F_{1}-Q F_{2}>k$. 
Sejam $\lambda$ a circunferência de centro $F_{1}$ e raio $k$ e $A$ o ponto de interseção do segmento $F_{1} P$ com $\lambda$. Então, por construção, $P A=P F_{2}$ e $P$ pertence à mediatriz do segmento $A F_{2}$. Seja $r$ tal mediatriz, e queremos mostrar que $r$ é tangente à hipérbole no ponto $P$. Para isso, seja $Q \neq P$ sobre $r$. Assim,

$$
Q F_{2}=Q A
$$

e pela condição de existência do triângulo $\triangle F_{1} A Q$,

$$
Q F_{1}<F_{1} A+Q A=k+Q F_{2} \Longrightarrow Q F_{1}-Q F_{2}<k .
$$

Então toda $r$, com exceção de $P$, está contida na mesma região $R 1$ delimitada por $H$ e portanto $r$ é tangente a $H$. Veja a Figura 7.4 .

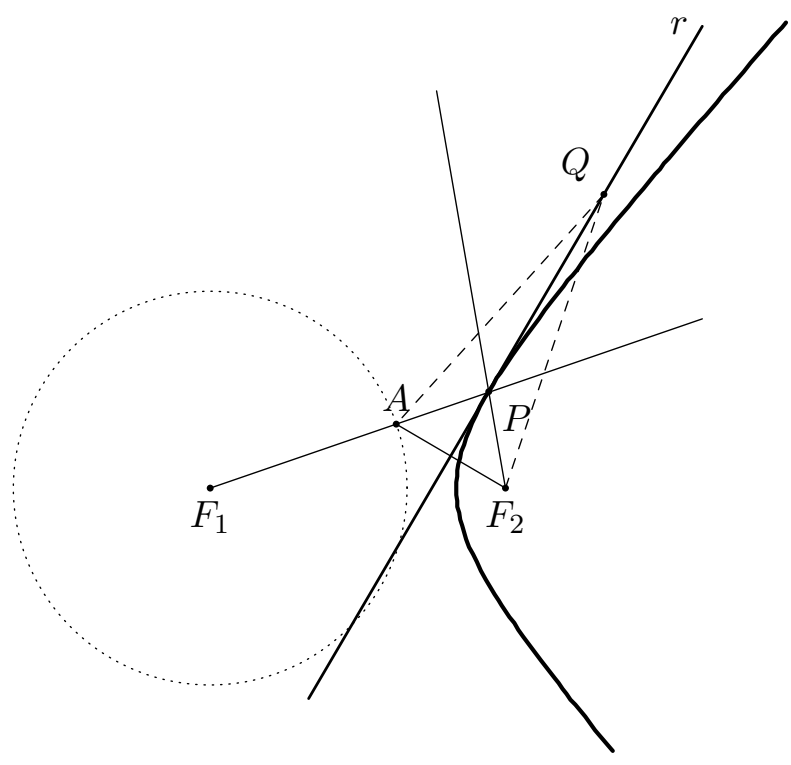

Figura 7.4: Reta tangente a hipérbole

Como $r$ é mediatriz de $A F_{2}$, os ângulos ente as retas $P F_{1}$ e $P F_{2}$ com $r$ são congruentes. Assim, se algum raio vier por uma das retas na direção de um dos focos, independente da região $R_{1}$ ou $R_{2}$ em que estiver, será refletido em $P$ na direção do outro foco, mantendo-se na mesma região. 


\subsection{Sistema de navegação LORAN}

Uma outra aplicação para hipérbole se encontra no sistema de navegação LORAN (Long Range Navigation), onde duas estações de rádio de localizações conhecidas emitem sinais ao mesmo tempo a um navio ou avião, e o computador de bordo calcula a diferença de tempo na recepção, transformando-a em diferença de distâncias. Assim, o navegador determina uma equação de hipérbole onde deve localizar-se, com focos nas estações. Com uma terceira estação, determina outra hipérbole. Logo, sua localização é determinada pela interseção entre tais hipérboles.

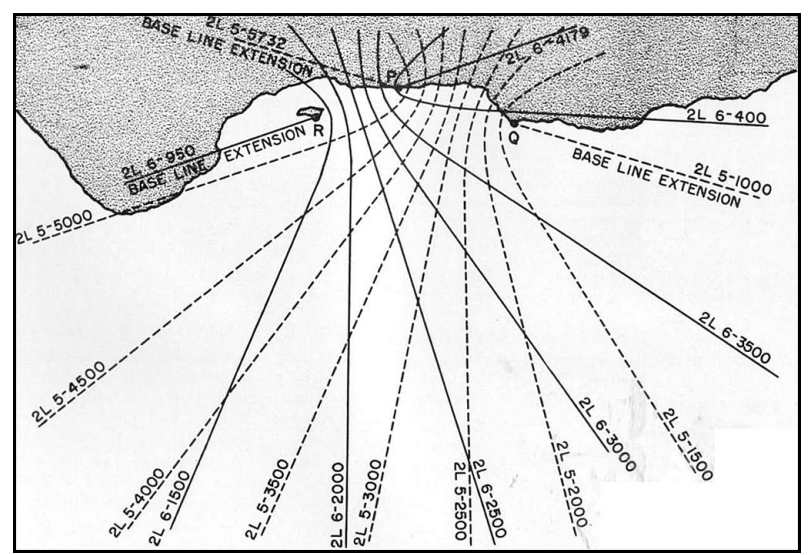




\section{Referências Bibliográficas}

[1] Ávila, G., A Hipérbole e os Telescópios, Revista do Professor de Matemática, n. 34 .

[2] Boyer, C., História da matemática. 2 $2^{\mathrm{a}}$ ed. São Paulo: Edgar Bluncher/Edusp, 1996.

[3] Boulos, P. / Camargo, I. de, Geometria Analítica: um tratamento vetorial. $3^{\text {a }}$ ed. São Paulo, PEARSON, ABDR editora afiliada.

[4] Conde, A., Geometria Analitica, Editora Atlas.

[5] Eves, H., Introdução à história da matemática. Campinas-SP: Unicamp, 1995.

[6] Garbi, G. G. A Rainha das Ciências. Um passeio histórico pelo maravilhoso mundo da Matemática. $2^{\mathrm{a}}$ Edição Aplicada. São Paulo: Editora Livraria da Física.

[7] Gonçalves, L., Propriedades Reflexivas das Cônicas. Dissertação de Mestrado. Pontifícia Universidade Católica do Rio de Janeiro (PUC-RJ). Março de 2014.

[8] Hop, D., Dandelin Spheres, disponível em http://www.clowder.net/ hop/Dandelin/Dandelin.html.

[9] Iezzi, G., Fundamentos de Matemática Elementar, Atual Editora, 5a edição.

[10] Lima, E. L., Geometria Analítica e Álgebra Linear, IMPA, $2^{\text {a }}$ edição. 
[11] Miranda, C. M., Construção de um mesa de bilhar elíptica como recurso motivacional para o estudo de cônicas no ensino médio. Dissertação de Mestrado. Universidade Estadual de Santa Cruz: Departamento de Ciências Exatas e Tecnológicas. Ilhéus-2013.

[12] Oliveira, Oswaldo Rio Branco de O., Cônicas (propriedades de reflexão), acesso em 19 de outubro de 2016, disponível em https://www.ime.usp. $\mathrm{br} /{ }^{\sim}$ oliveira/conicaspubli.pdf

[13] Proposta curricular do estado de São Paulo (2008)/ Coord. Maria Inês Fini - São Paulo: SEE, 2008

1.Matemática (ensino fundamental e médio - estudo e ensino. I. Fini, Maria Inês. II. São Paulo (estado) Secretaria da Educação.

CDD22ed. 510.7

[14] Sato, Jocelino, Fórmula de redução e classificação de uma cônica, acesso em 30 de setembro de 2016, disponível em http://www . sato .prof .ufu. $\mathrm{br} /$ Conicas/node12.html.

[15] Silva, G. S., Por que elipse, parábola e hipérbole?, Revista do professor de matemática, n. 7 .

[16] Simões, George. Cálculo com geometria analítica. Vol 2. São Paulo: Mc Graw Hill, 1985.

[17] Souza Jr., J. C. e Cardoso, Andréa, Estudo das cônicas com Geometria Dinâmica, Revista do Professor de Matemática, n. 68.

[18] Valladares, Renato J. C., Elipse, Sorrisos e Sussurros, Revista do Professor de Matemática, Revista do Professor de Matemática, n. 36.

[19] Venturi J. J., O segundo problema clássico da Geometria: a duplicação do cubo, acesso em 11 de dezembro de 2015, disponível em: www.educacional.com.br/articulistas/outrosOutros_ artigo . asp?artigo=artigo0057

[20] Wagner, E., Por que as antenas são parabólicas, Revista do Professor de Matemática, n. 33. 